This is an electronic reprint of the original article. This reprint may differ from the original in pagination and typographic detail.

Author(s): Myöhänen, Petri; Tuovinen, Riku; Korhonen, Topi; Stefanucci, G.; van Leeuwen, Robert

Title: $\quad$ Image charge dynamics in time-dependent quantum transport

Year: $\quad 2012$

Version:

Please cite the original version:

Myöhänen, P., Tuovinen, R., Korhonen, T., Stefanucci, G., \& van Leeuwen, R. (2012). Image charge dynamics in time-dependent quantum transport. Physical Review $B$, 85(7). https://doi.org/10.1103/PhysRevB.85.075105

All material supplied via JYX is protected by copyright and other intellectual property rights, and duplication or sale of all or part of any of the repository collections is not permitted, except that material may be duplicated by you for your research use or educational purposes in electronic or print form. You must obtain permission for any other use. Electronic or print copies may not be offered, whether for sale or otherwise to anyone who is not an authorised user. 


\title{
Image charge dynamics in time-dependent quantum transport
}

\author{
Petri Myöhänen, ${ }^{1}$ Riku Tuovinen, ${ }^{1}$ Topi Korhonen, ${ }^{1}$ Gianluca Stefanucci, ${ }^{2,3,4}$ and Robert van Leeuwen ${ }^{1,4}$ \\ ${ }^{1}$ Department of Physics, Nanoscience Center, FI-40014, University of Jyväskylä, Jyväskylä, Finland \\ ${ }^{2}$ Dipartimento di Fisica, Università di Roma Tor Vergata, Via della Ricerca Scientifica 1, IT-00133 Rome, Italy \\ ${ }^{3}$ Laboratori Nazionali di Frascati, Istituto Nazionale di Fisica Nucleare, Via E. Fermi 40, IT-00044 Frascati, Italy \\ ${ }^{4}$ European Theoretical Spectroscopy Facility $(E T S F)$
}

(Received 24 November 2011; revised manuscript received 14 January 2012; published 7 February 2012)

\begin{abstract}
In this work, we investigate the effects of the electron-electron interaction between a molecular junction and the metallic leads in time-dependent quantum transport. We employ the recently developed embedded Kadanoff-Baym method [Phys. Rev. B 80, 115107 (2009)] and show that the molecule-lead interaction changes substantially the transient and steady-state transport properties. We first show that the mean-field Hartree-Fock (HF) approximation does not capture the polarization effects responsible for the renormalization of the molecular levels neither in nor out of equilibrium. Furthermore, due to the time-local nature of the HF self-energy, there exists a region in parameter space for which the system does not relax after the switch-on of a bias voltage. These and other artifacts of the HF approximation disappear when including correlations at the second-Born or GW levels. Both these approximations contain polarization diagrams, which correctly account for the screening of the charged molecule. We find that by changing the molecule-lead interaction, the ratio between the screening and relaxation time changes, an effect which must be properly taken into account in any realistic time-dependent simulation. Another important finding is that while in equilibrium the molecule-lead interaction is responsible for a reduction of the highest occupied molecular orbital-lowest unoccupied molecular orbital (HOMO-LUMO) gap and for a substantial redistribution of the spectral weight between the main spectral peaks and the induced satellite spectrum, in the biased system it can have the opposite effect, i.e., it sharpens the spectral peaks and opens the HOMO-LUMO gap.
\end{abstract}

DOI: 10.1103/PhysRevB.85.075105

PACS number(s): 72.10.Bg, 71.10.-w, 73.63.-b, 85.30.Mn

\section{INTRODUCTION}

The electron transport through molecular devices has gained remarkable interest during the last years, primarily due to experimental advances in creating conductive moleculemetal junctions. ${ }^{1,2}$ From the experimental point of view, these systems are very attractive for their potential utilization as the next-generation nanometer-scale building blocks for future integrated circuits exceeding up to terahertz operating frequencies. For theorists, the experimental realization of electron transport through molecules opens up a new intriguing and challenging playground for both theoretical and numerical modeling of the underlying physical processes. Understanding these processes at a microscopic level is crucial for the future development of molecular electronics.

Considerable progress has been made to investigate both steady-state ${ }^{3-13}$ and time-dependent ${ }^{14-17,19-32}$ transport properties of metal-nanostructure-metal junctions. As an increasing trend, the system is partitioned into an explicitly treated interacting region coupled to noninteracting electron reservoirs (leads), which act as source and sink terminals. However, the partitioning into an interacting and a noninteracting part is, in general, not well justified due to the long-range nature of the Coulomb interaction. Recently, there have been some advances in calculating transport properties of nanoscale junctions while incorporating the electron-elecron interaction in the leads. Perfetto et al. ${ }^{33}$ recently found that by modeling the electron-electron interaction in low-dimensional leads with the Luttinger model, the initial correlation effects are not washed out in the long-time limit and contribute substantially to the steady-state current. Bohr et al. ${ }^{34}$ and Borda et al..$^{35}$ investigated the effects of the lead-molecule interactions in the interacting resonant level model and showed that it can lead to a strong enhancement of the conductance. More recently, these studies have been extended to long-range lead-molecule interactions. ${ }^{36,37}$

Considerable attention has also been devoted to the effects of surface polarization (or image charge formation). In Refs. 38-42, it was shown that polarization effects can dramatically change the quasiparticle gap of molecules near the metallic surfaces where the dynamical correlation effects and molecule-lead hopping integrals reduce the molecular energy gap across the binding regime from gas phase to physisorption. Clearly, this renormalization of the molecular levels can have a large impact on the transport properties of weakly coupled molecular junctions. Yet, the question of how the molecule-lead interactions and, consequently, the formation of an image charge affects the ultrafast electron dynamics before a steady state (if any) is reached is still unanswered. This paper wants to address two fundamental issues: What is the time scale to screen molecular charge fluctations induced by the sudden switch-on of an external bias? What are the scattering processes (or Feynman diagrams) relevant for an accurate description of the screening and relaxation dynamics?

To answer these questions, we will use the KadanoffBaym method, which has recently been applied to both finite isolated ${ }^{43-47}$ and quantum transport systems ${ }^{16-18}$ and has the merit of preserving all basic conservation laws. ${ }^{48,49} \mathrm{We}$ show that the mean-field Hartree-Fock (HF) approximation suffers from several limitations in this context. Aside from being unable to account for dynamical polarization effects, the Hartree-Fock approximation can give rise to "unstable" time-dependent solutions with persistent oscillations in density 
and current. All mean-field artifacts disappear when including polarization effects in the self-energy, either at the secondBorn or GW level. These correlated solutions have recently been assessed in the Anderson model, ${ }^{50}$ and good agreement with time-dependent density matrix renormalization group (DMRG) data was found. ${ }^{51}$ Here, we employ them for a thorough analysis of the screening versus relaxation dynamics as a function of the interaction strength, the molecule-lead hopping integrals, and the external bias. We find that the relaxation time $\tau_{\text {rel }}$ becomes shorter when increasing the molecule-lead interactions at the second-Born and GW levels, while the screening time $\tau_{\text {scr }}$ is roughly independent on the interaction strength. Often, the time-dependent quantum transport simulations are based on the assumption that $\tau_{\text {scr }} / \tau_{\text {rel }} \ll$ 1. Our results show that the molecule-lead interaction can substantially increase this ratio. Another remarkable effect of the molecule-lead interaction is that for large enough biases, the electronic correlations can sharpen the spectral peaks and widen the gap between the levels of highest occupied molecular orbital (HOMO) and lowest unoccupied molecular orbital (LUMO). This behavior is exactly the opposite of the equilibrium behavior and indicates that in the presence of a current flow, the screening lengthens the HOMO-LUMO quasiparticle lifetime and decreases (increases) the ionization potential (electron affinity).

The paper is organized as follows. In Sec. II, we introduce the model Hamiltonian for quantum transport simulations and discuss the exact solution for zero molecule-lead hopping integrals. We also give a short account of the theoretical background and defer the reader to previously published work for details. In Sec. III, we analyze the screening versus relaxation time and the effect of the formation of an image charge in the equilibrium spectral function. Section IV deals with the short-time dynamics of the lead-molecule-lead junction driven out of equilibrium by the sudden switch-on of a constant bias, while Sec. V deals with the long-time dynamics, and in particular with the absence of relaxation within $\mathrm{HF}$ and the effects of screening in the $I-V$ characteristic. The main conclusions are then drawn in Sec. VI.

\section{IMAGE CHARGE MODEL}

\section{A. Hamiltonian}

To study the image charge effect, we consider a model Hamiltonian that was introduced in Refs. 39 and 40. This image charge model Hamiltonian is displayed schematically in Fig. 1 and reads as

$$
\hat{H}(t)=\hat{H}_{\mathrm{mol}}+\hat{H}_{\mathrm{ch}}(t)+\hat{V}-\mu \hat{N},
$$

where $\mu$ is the chemical potential and $\hat{N}$ is the operator for the total number of particles. The molecular region is modeled by a two-level system representing the highest occupied molecular orbital $(H)$ and the lowest unoccupied molecular orbital $(L)$ with energies $\epsilon_{H}$ and $\epsilon_{L}$, respectively:

$$
\begin{aligned}
\hat{H}_{\mathrm{mol}}= & \epsilon_{H} \hat{n}_{H}+\epsilon_{L} \hat{n}_{L} \\
& +U_{0}\left(\hat{n}_{H \uparrow} \hat{n}_{H \downarrow}+\hat{n}_{L \uparrow} \hat{n}_{L \downarrow}\right)+U_{H L} \hat{n}_{H} \hat{n}_{L} .
\end{aligned}
$$

The interaction strengths $U_{0}$ and $U_{H L}$ account for the intralevel and interlevel electron repulsion. Furthermore, we used the

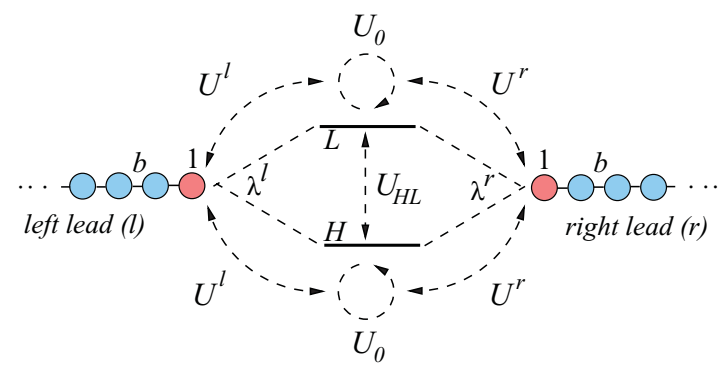

FIG. 1. (Color online) Image charge model for quantum transport.

standard notation $\hat{n}_{i}=\sum_{\sigma=\uparrow \downarrow} \hat{n}_{i \sigma}$ for the particle number operator of the molecular level $i=H, L$, where $\hat{n}_{i \sigma}=\hat{c}_{i \sigma}^{\dagger} \hat{c}_{i \sigma}$ and $\hat{c}_{i \sigma}^{\dagger}$ and $\hat{c}_{i \sigma}$ are the electron creation and annihilation operators.

The second term in Eq. (1) describes the left $(\alpha=l)$ and right $(\alpha=r)$ leads

$$
\hat{H}_{\mathrm{ch}}(t)=\sum_{\alpha=l, r} \sum_{i, j=1}^{\infty} \sum_{\sigma=\uparrow \downarrow}\left[h_{i j}^{\alpha}+\delta_{i j} W^{\alpha}(t)\right] \hat{c}_{\alpha i \sigma}^{\dagger} \hat{c}_{\alpha j \sigma},
$$

which are modeled as one-dimensional semi-infinite tightbinding (TB) chains subject to time-dependent uniform bias voltages $W^{\alpha}(t)$. The TB parameters $h_{i j}$ of the chain are chosen so that $h_{i j}=b$ for $i, j$ nearest neighbors and zero otherwise. Finally, $\hat{c}_{\alpha i \sigma}^{\dagger}$ and $\hat{c}_{\alpha j \sigma}$ are the creation and annihilation operators for electrons in lead $\alpha$, site $i=1,2, \ldots, \infty$, and $\operatorname{spin} \sigma$.

The third term in Eq. (1) describes the interaction between the molecular levels and the TB chains:

$$
\begin{aligned}
\hat{V}= & \sum_{\alpha=l, r} \sum_{i=H, L} \sum_{\sigma=\uparrow \downarrow} \lambda^{\alpha}\left(\hat{c}_{\alpha 1 \sigma}^{\dagger} \hat{c}_{i \sigma}+\hat{c}_{i \sigma}^{\dagger} \hat{c}_{\alpha 1 \sigma}\right) \\
& +\sum_{\alpha=l, r} U^{\alpha}\left(\hat{n}_{\alpha 1}-1\right)\left(\hat{N}_{\mathrm{mol}}-2\right) .
\end{aligned}
$$

Here, $\lambda^{\alpha}$ and $U^{\alpha}$ are the hopping integrals (proportional to the hybridization of the molecular levels) and Coulomb interaction strengths between the HOMO and LUMO levels and the terminal site of lead $\alpha$. The quantity $\hat{n}_{\alpha 1}$ is the particle number operator of site 1 of lead $\alpha, \hat{n}_{\alpha 1}=\sum_{\sigma=\uparrow \downarrow} \hat{c}_{\alpha 1 \sigma}^{\dagger} \hat{c}_{\alpha 1 \sigma}$, while $\hat{N}_{\text {mol }}$ is the total number of particle operator of the molecule $\hat{N}_{\text {mol }}=\hat{n}_{H}+\hat{n}_{L}$. We consider the system initially in equilibrium at zero temperature, zero bias, $W^{\alpha}=0$, and at half-filling. Then, the average density on the lead sites is unity, while the average density of the HOMO and LUMO levels is 2 and 0 , respectively. To guarantee the charge neutrality of the interacting region, we subtracted a positive background charge of 1 from $\hat{n}_{\alpha 1}$ and of 2 from $\hat{N}_{\text {mol }}$.

This completes the explanation and justification of the image charge model (ICM). It can be considered as an extension of the interacting resonant level model to study molecular excitons and polarization effects. The ICM can, of course, be further refined by including interactions in the leads and a direct lead-lead interaction, and can be further generalized to two- or three-dimensional leads, more molecular levels, etc. Equation (1), however, provides the minimal model to study the effects of image charges in the nonequilibrium properties 
of nanoscale junctions, and in this paper we will not discuss any of the aforementioned extensions.

\section{B. Uncontacted case: Exact solution}

The ICM can be solved exactly for zero hybridization, i.e., $\lambda^{r}=\lambda^{l}=0$, since in this case the operators $\hat{n}_{H}$ and $\hat{n}_{L}$ commute with the Hamiltonian and hence the number of electrons on the $H$ and $L$ levels are conserved quantities. Let us consider for simplicity the unperturbed Hamiltonian $\hat{H}$ obtained from Eq. (1) by setting the bias $W^{\alpha}$ to zero. All eigenstates of $\hat{H}$ have the form

$$
|M, s\rangle=\prod_{j} \theta_{j} \hat{c}_{j}^{\dagger}\left|\Phi_{s}\right\rangle
$$

Here, the $\hat{c}_{j}^{\dagger}$ operators create electrons on the molecular level $j \in\{H \uparrow, H \downarrow, L \uparrow, L \downarrow\}$, and $\theta_{j}$ is either equal to one or zero depending on what states one likes to occupy. The corresponding molecular configuration is specified by the collective quantum number $M$. The state $\left|\Phi_{s}\right\rangle$ is the tensor product of the vacuum state of the molecule and the $s$ th excited state of the uncontacted leads and has the property $\hat{n}_{j}\left|\Phi_{s}\right\rangle=0$. For example, a state with two electrons in the HOMO level of the molecule is $|H \uparrow, H \downarrow, s\rangle=c_{H \uparrow}^{\dagger} c_{H \downarrow}^{\dagger}\left|\Phi_{s}\right\rangle$. To find the secular equation for the $\left|\Phi_{s}\right\rangle$, we apply $\hat{H}$ to $|M, s\rangle$ and find

$$
\begin{aligned}
\hat{H} & |M, s\rangle \\
& =\left[\hat{H}_{\mathrm{mol}}+\hat{H}_{\mathrm{ch}}+\sum_{\alpha} U^{\alpha}\left(\hat{n}_{\alpha 1}-1\right)\left(\hat{N}_{\mathrm{mol}}-2\right)\right]|M, s\rangle \\
& =\left[E_{M}+\mathcal{E}_{M, s}\right]|M, s\rangle=E_{M, s}|M, s\rangle
\end{aligned}
$$

where $E_{M}$ is the total energy of the isolated molecule with $N_{\text {mol }}$ electrons satifying the eigenvalue equation

$$
\hat{H}_{\mathrm{mol}}|M, s\rangle=E_{M}|M, s\rangle,
$$

while $\mathcal{E}_{M, s}$ is the total energy of the uncontacted leads in the presence of the potential $U^{\alpha}\left(N_{\text {mol }}-2\right)$ at the terminal sites

$$
\begin{aligned}
& \hat{H}_{\mathrm{ch}}(U)|M, s\rangle \\
& \quad \equiv\left[\hat{H}_{\mathrm{ch}}(U=0)+\sum_{\alpha} U^{\alpha}\left(N_{\mathrm{mol}}-2\right)\left(\hat{n}_{\alpha 1}-1\right)\right]|M, s\rangle \\
& \quad=\mathcal{E}_{M, s}|M, s\rangle .
\end{aligned}
$$

This potential depends on the strength of the Coulomb interaction $U^{\alpha}$ and on the number $N_{\text {mol }}$ of electrons on the molecule. Once we know the electronic configuration of the molecule, the problem reduces to solving the eigenvalue equation (8) for a noninteracting TB chain with an impuritylike potential at the terminal site. If the molecule is charge neutral, $N_{\text {mol }}=2$, this potential is zero. However, adding (removing) an electron from the charge-neutral molecule gives rise to a potential $+U^{\alpha}\left(-U^{\alpha}\right)$. This, in turn, causes a depletion (accumulation) of charge, which is exactly the image charge.

It is worth stressing that the presence of the lead-molecule interaction affects the total energies of the charged system [see again Eq. (8)], and consequently changes the addition and removal energies. Consider, for instance, the solution for a simple two-site chain and a lead-molecule interaction
$U^{r}=U$ and $U^{l}=0$ (no coupling to the left lead). It is easy to show that the electron affinity is $A=\epsilon_{L}+2 U_{H L}+2|b|-$ $2 \sqrt{(U / 2)^{2}+b^{2}}$, while the ionization energy is $I=\epsilon_{H}+U_{0}-$ $2|b|+2 \sqrt{(U / 2)^{2}+b^{2}}$ (see Appendix A). The difference $A-I$ reduces with increasing $U$ and the quasiparticle gap collapses. This can also be viewed from another, more general, point of view. Consider for simplicity that $U^{\alpha}=U$ for both leads and that the intramolecular interactions $U_{0}$ and $U_{H L}$ are zero. If the molecule is charge neutral $\left(N_{\text {mol }}=2\right)$, the energies of the $N$ and $N \pm 1$ particle ground states (with the constraint that the electron is added to or removed from the molecule) are given by

$$
\begin{gathered}
E_{N}=2 \epsilon_{H}+\mathcal{E}_{\mathrm{GS}}(0), \\
E_{N+1}=2 \epsilon_{H}+\epsilon_{L}+\mathcal{E}_{\mathrm{GS}}(U), \\
E_{N-1}=\epsilon_{H}+\mathcal{E}_{\mathrm{GS}}(-U),
\end{gathered}
$$

where we defined $\mathcal{E}_{\mathrm{GS}}(U)$ to be the ground-state energy of the Hamiltonian $\hat{H}_{\mathrm{ch}}(U)$ of Eq. (8). Therefore, the electron affinity $A$ and ionization energy $I$ read as

$$
\begin{gathered}
A=E_{N+1}-E_{N}=\epsilon_{L}+\mathcal{E}_{\mathrm{GS}}(U)-\mathcal{E}_{\mathrm{GS}}(0), \\
I=E_{N}-E_{N-1}=\epsilon_{H}+\mathcal{E}_{\mathrm{GS}}(0)-\mathcal{E}_{\mathrm{GS}}(-U) .
\end{gathered}
$$

Let $\left|\Phi_{\mathrm{GS}}(u)\right\rangle$ be the ground state of $\hat{H}_{\mathrm{ch}}(U)$. Then, according to the Hellman-Feynman theorem, ${ }^{52}$

$$
\frac{d \mathcal{E}_{\mathrm{GS}}(u)}{d u}=\left\langle\Phi_{\mathrm{GS}}(u)\left|\frac{d \hat{H}_{\mathrm{ch}}(u)}{d u}\right| \Phi_{\mathrm{GS}}(u)\right\rangle
$$

and, therefore,

$$
\mathcal{E}_{\mathrm{GS}}(U)-\mathcal{E}_{\mathrm{GS}}(0)=\sum_{\alpha} \int_{0}^{U}\left[n_{\alpha 1}(u)-1\right] \mathrm{d} u .
$$

From this equation, we see clearly how the ground-state energy depends on the molecular occupation: If we add an electron to the molecule, we push away charge from the first sites of the leads and, hence, the integral is negative and the affinity lowers. On the other hand, if we remove an electron from the molecule, we attract charge to the first sites of the leads and the ionization energy increases.

The bottom panel of Fig. 2 shows how the image charge is built up in the lead. We plot the time evolution of the density at the first site of a semi-infinite chain when the impuritylike potential $U=0.5$ is suddenly switched on at time $t=0$ on site 1 . The different curves correspond to different hopping parameters in the lead $b=$ $-0.5,-1.0,-1.5,-2.0$. By increasing $b$, the frequency of the transient oscillations increases and the steady state is reached faster. This behavior can be easily understood by inspecting the imaginary part of the density response function $\chi_{11}(\omega)$ (top right panel of Fig. 2). In Appendix B, we show that this quantity has a maximum at $\omega \sim 2|b|$, which corresponds to the oscillation frequency of the density. The width of the maximum grows like $2|b|$ and its inverse gives the screening time, i.e., the time scale for the image charge formation. Furthermore, from the top left panel, we see that $\chi_{11}(\omega=0)$ behaves as $1 / b$, which is consistent with the larger induced charge in the long-time limit. 

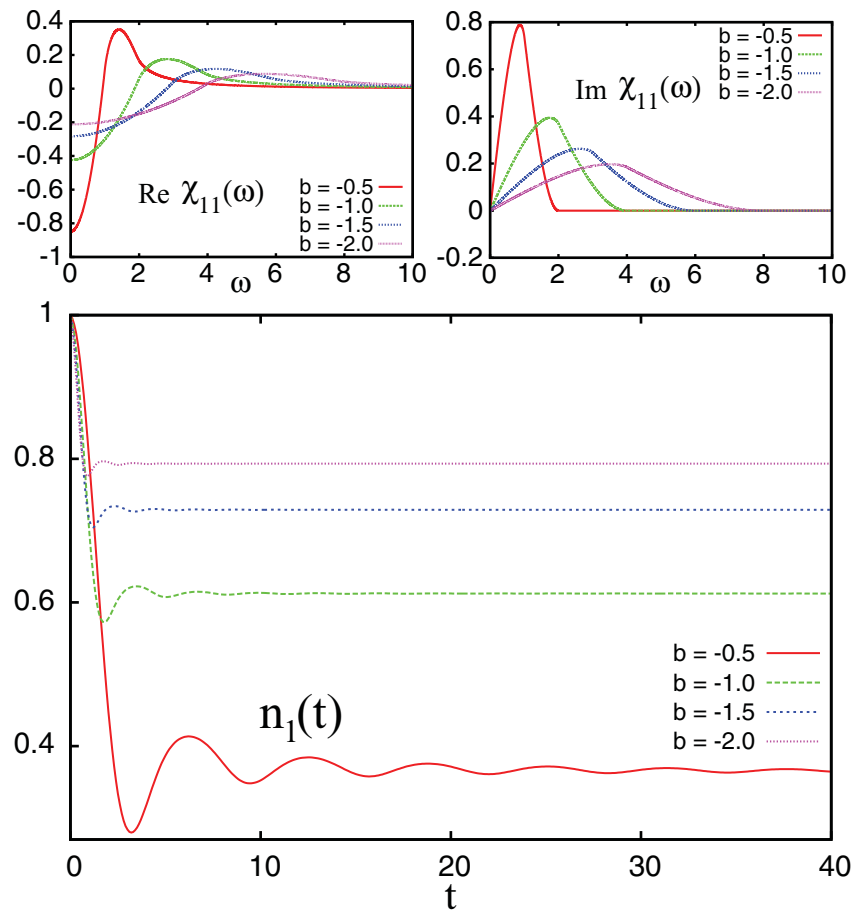

FIG. 2. (Color online) Top left and right panels: The real and imaginary parts of the dynamical response function. Bottom panel: The electron density at the terminal site of the TB chain as a function of time when the impurity potential $U=0.5$ is suddenly switched on. The different curves correspond to different values of the hopping parameter $b=-0.5,-1.0,-1.5,-2.0$.

\section{Many-body treatment}

The ICM does not have an exact solution for the contacted case, and to study it both in and out of equilibrium, we use the nonequilibrium Green's function (NEGF) method based on time propagation of the embedded Kadanoff-Baym equations. ${ }^{16,17,48,53}$ The basic quantity in the formalism is the one-particle Green's function

$$
G_{k l}\left(z, z^{\prime}\right)=-\mathrm{i} \delta_{\sigma_{k} \sigma_{l}} \frac{\operatorname{Tr}\left\{\mathcal{T}\left[e^{-i \int_{c} \mathrm{~d} \bar{z} \hat{H}(\bar{z})} \hat{c}_{k \sigma_{k}}(z) \hat{c}_{l \sigma_{l}}^{\dagger}\left(z^{\prime}\right)\right]\right\}}{\operatorname{Tr}\left\{e^{-i \int_{c} \mathrm{~d} \bar{z} \hat{H}(\bar{z})}\right\}},
$$

where we used the notations $\hat{c}_{k \sigma_{k}}$ and $\hat{c}_{l \sigma_{l}}^{\dagger}$ to denote electron annihilation and creation operators either in the molecule or in the leads. Note that since the Hamiltonian is invariant under rotations in spin space (along the entire Keldysh contour), then the Green's function is diagonal in the spin indices and independent of the spin component. We therefore use only the orbital indices to specify its matrix elements. In the above definition, $z, z^{\prime}$ are the time indices on the Keldysh contour $c, \mathcal{T}$ is the time-ordering operator on the Keldysh contour, and $\operatorname{Tr}\{\ldots\}$ signifies the trace over the Fock space of all many-body states. The Green's function $G$ is the solution of the integrodifferential equation of motion on the Keldysh contour

$$
\left[\mathrm{i} \partial_{z}-h(z)\right] G\left(z, z^{\prime}\right)=\delta\left(z, z^{\prime}\right)+\int_{c} d \bar{z} \Sigma[G](z, \bar{z}) G\left(\bar{z}, z^{\prime}\right)
$$

where $h(z)$ is the Hamiltonian in the one-particle Hilbert space, $\delta\left(z, z^{\prime}\right)$ is the contour delta function, and $\Sigma[G]$ is the selfenergy kernel containing all the information on the manybody and embedding effects. ${ }^{16,17}$ For the purpose of a practical implementation of the Hamiltonian (1), we divide the system into interacting $(C)$ and noninteracting $(\alpha)$ regions and write the single-particle part and the interaction part of $C$ as [see Eq. (1)] $[h]_{i j}(t)=\left(\begin{array}{ccc}-2 U^{l}+W^{l}(t) & \lambda^{l} & \lambda^{l} \\ \lambda^{l} & \epsilon_{H}-U^{l}-U^{r} & 0 \\ \lambda^{l} & 0 & \epsilon_{L}-U^{l}-U^{r} \\ 0 & \lambda^{r} & \lambda^{r}\end{array}\right.$

Using this notation, the Hamiltonian (1) transforms into

$$
\begin{aligned}
\hat{H}= & \sum_{i j \in C} \sum_{\sigma=\uparrow \downarrow} h_{i j}(t) \hat{c}_{i \sigma}^{\dagger} \hat{c}_{j \sigma}+\frac{1}{2} \sum_{i j \in C} \sum_{\sigma=\uparrow \downarrow} v_{i j} \hat{c}_{i \sigma}^{\dagger} \hat{c}_{j \sigma}^{\dagger} \hat{c}_{j \sigma} \hat{c}_{i \sigma} \\
& +\sum_{\alpha=l, r} \sum_{i j \in \alpha} \sum_{\sigma=\uparrow \downarrow}\left[h_{i j}^{\alpha}+\delta_{i j} W^{\alpha}(t)\right] \hat{c}_{\alpha i \sigma}^{\dagger} \hat{c}_{\alpha j \sigma}-\mu \hat{N},
\end{aligned}
$$

where $C$ contains the molecular levels and also the terminal sites of the leads subjected to the bias voltages $W^{\alpha}(t)$. Furthermore, $\alpha=L, R$ are the noninteracting parts of the left and right leads. We choose $U_{0}=U_{H L}=U_{L H}=1, \mu=0$, $\epsilon_{H}=-2$, and $\epsilon_{L}=-1$ throughout the rest of this paper.

We will solve Eq. (17) with a Hartree-Fock, second-Born (2B), and GW many-body self-energy. The quality of the

$$
\left.\begin{array}{c}
0 \\
\lambda^{r} \\
\lambda^{r} \\
-2 U^{r}+W^{r}(t)
\end{array}\right), \quad[v]_{i j}=\left(\begin{array}{cccc}
0 & U^{l} & U^{l} & 0 \\
U^{l} & U_{0} & U_{H L} & U^{r} \\
U^{l} & U_{L H} & U_{0} & U^{r} \\
0 & U^{r} & U^{r} & 0
\end{array}\right) .
$$

2B and GW self-energy has recently been assessed in the Anderson model ${ }^{50}$ by comparing the time-dependent current and density against time-dependent DMRG results. ${ }^{51}$ Good agreement was found in the parameter regime that we discuss below.

It is instructive and useful for our later analysis to discuss the many-body approximations in the uncontacted case. However, we stress that in the results section, we used many-body selfenergies evaluated at the fully contacted and self-consistent Green's functions. Since the number of electrons in the $H$ and $L$ levels are conserved quantities in the uncontacted case, the Green's function $G_{H k}=\delta_{H k} G_{H H}$ and similarly $G_{L k}=\delta_{L k} G_{L L}$ for all levels and sites $k$ of the system. The HF approximation consists of the first two diagrams in Fig. 3(a). 
(a)
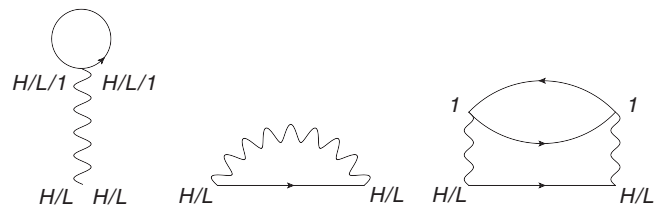

(b)
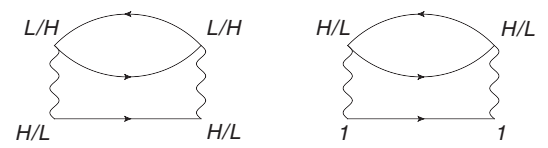

FIG. 3. Self-energy diagrams for $\lambda^{l}=\lambda^{r}=0$. (a) The only nonzero diagrams of the GW self-energy. (b) Second-order selfenergy diagrams that are zero. All higher-order diagrams are also zero.

The labels of the vertices refer to the molecular levels $H$, $L$, and the terminal site of lead $\alpha$ (which is simply denoted by 1 independently of $\alpha$ ). The 2B self-energy is obtained by adding to the HF self-energy the first bubble diagram and the second-order exchange diagram. For zero hybridization, however, the second-order exchange diagram vanishes since it either contains an off-diagonal element of the Green's function (which is zero) or a product of lesser and greater diagonal element of the molecular Green's functions, e.g., $G_{H H}^{>} G_{H H}^{<}$. Having the equilibrium system with two electrons in $H$ and zero in $L$, it must be $G_{L L}^{<}=G_{H H}^{>}=0$. Consequently, only the first bubble diagram survives and the 2B self-energy diagrams are all displayed in Fig. 3(a). Note that if the external vertices of the bubble self-energy diagram lie on the terminal site of the leads [second diagram of Fig. 3(b)], the diagram vanishes. This is a direct consequence of the fact that the polarization diagram with $H$ or $L$ as vertices is proportional to the product $G_{H H}^{>} G_{H H}^{<}$or $G_{L L}^{>} G_{L L}^{<}$, which is zero. For the same reason, the first diagram of Fig. 3(b) is also zero. The physical origin of this result is that one can not create particle-hole excitations on the molecules without changing the number of electrons in the $H / L$ levels. The many-body self-energy in the $\mathrm{GW}$ approximation is $\Sigma=i \mathrm{GW}$ where the screened interaction $\mathrm{W}$ is approximated as a geometric series of bare polarization diagrams connected by interaction lines. Since the only bare polarization diagram is the particle-hole propagator going from 1 to 1 , the GW approximation coincides with the $2 \mathrm{~B}$ approximation. In our ICM, there is no direct interaction between two electrons on the terminal site of the leads. It is therefore reasonable to expect that the $2 \mathrm{~B}$ and $\mathrm{GW}$ approximations perform similarly for small hybridizations. As we shall see in the next section, this expectation is indeed confirmed by our numerical calculations.

\section{COMPETING TIME SCALES AND SPECTRAL PROPERTIES}

In the previous section, we have seen that there is a characteristic screening time to build up charge after the addition or removal of an electron to or from the molecule. In the case that the molecule is contacted to the leads, there is another time scale that plays a role. This is the relaxation time to disperse the excess charge on the molecule into the leads. It

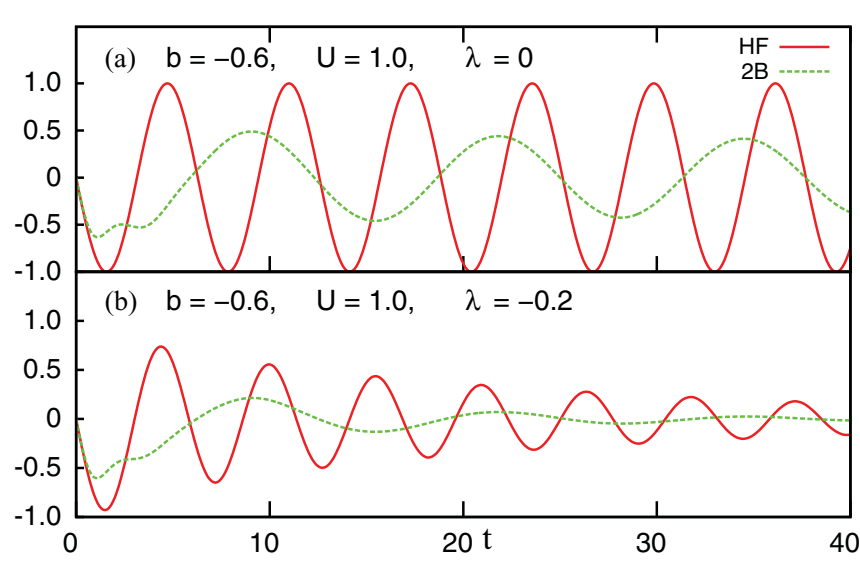

FIG. 4. (Color online) Green's function $G_{L L}^{>}(t, 0)$ for (a) HF and $2 \mathrm{~B}$ with $b=-0.6, U=1$, and $\lambda=0$, (b) HF and $2 \mathrm{~B}$ with $b=-0.6$, $U=1.0$, and $\lambda=-0.2$.

is the ratio between the screening time and the relaxation time that tells us how the system behaves under nonequilibrium conditions. The aim of this section is to extract these time scales from the equilibrium properties of the contacted system and to analyze the effects of the screening on the equilibrium spectral function. This will help us to gain insight in the more complicated case of quantum transport discussed in the next section. The analysis will be carried on using many-body Green's function methods since the contacted case is no longer analytically solvable.

\section{A. Screening and relaxation times}

In this section, we study the response of the system to the sudden addition or removal of an electron on the molecule within the $\mathrm{HF}, 2 \mathrm{~B}$, and $\mathrm{GW}$ approximations. The response of an added electron is encoded into the $G^{>}$and $G^{<}$Green's functions, which we can calculate within these many-body approximations both in real time and in frequency space. For instance, the LUMO Green's function $G_{L L}^{>}(t, 0)=$ $-i\left\langle\hat{c}_{L}(t) \hat{c}_{L}^{\dagger}(0)\right\rangle$ gives the probability amplitude of finding a particle on the LUMO level at time $t$ after being created at time 0 . In Fig. 4, we plot the real part of this quantity. This Green's function oscillates with a characteristic frequency equal to the addition energy of an electron to the LUMO level. In Fig. 4(a), we compare the $\mathrm{HF}$ and $2 \mathrm{~B}$ results for $\lambda^{l}=\lambda^{r}=0, U^{l}=0$, $U^{r}=U=1$, and $b=-0.6$. The correlated $2 \mathrm{~B}$ curve exhibits a short transient with a characteristic time scale $\sim 1 / b$. This transient has to be attributed to the buildup of the image charge, and its duration is consistent with the previous analysis of Fig. 2. Note that no transient is visible in the HF approximation, which therefore fails to describe the formation of the image charge. The interplay between the screening time and the relaxation time can be investigated by contacting the molecule to the leads. In Fig. 4(b), we consider the same parameters as in Fig. 4(a) except for $\lambda^{r}=\lambda=-0.2$, i.e., the molecule is contacted to the right lead. The main difference to the previous case is that both the HF and $2 \mathrm{~B}$ curves are damped (relaxation). Similarly to the uncontacted case, there is no evidence of screening in the HF approximation.

Let us now address in more detail the dependence of the relaxation time on the molecule-lead interaction $U$. In most 

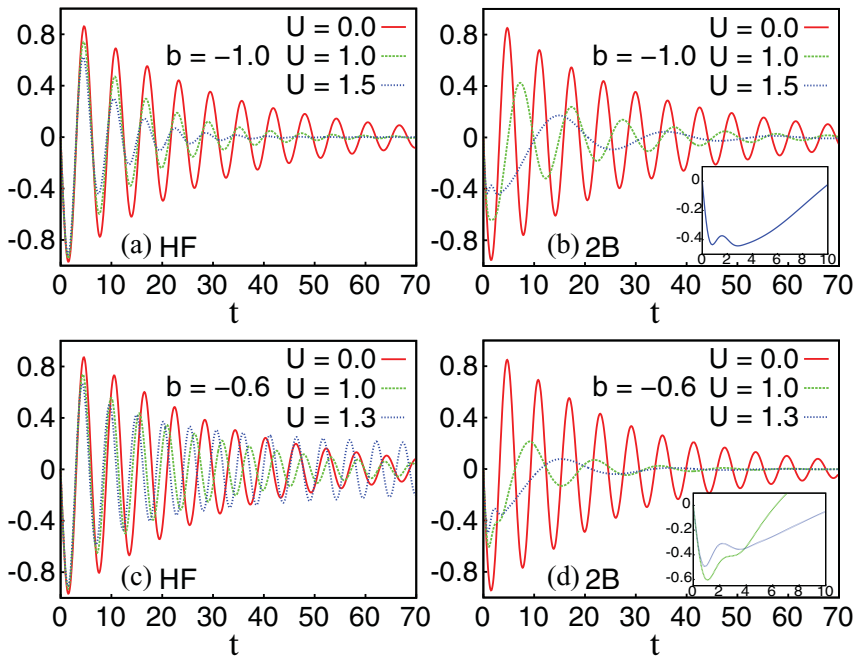

FIG. 5. (Color online) Green's function $G_{L L}^{>}(t, 0)$ for HF and 2B with $b=-1.0[(\mathrm{a})$ and (b)] and $b=-0.6[(\mathrm{c})$ and (d) $]$ and $\lambda=-0.2$ and different values of $U$.

physical situations, the bandwidth of the metallic leads is much larger than the molecule-lead coupling $b \gg \lambda$. Then, for small values of $U$, the relaxation time is proportional to $\tau_{\text {rel }} \sim \Gamma^{-1} \sim|b| / \lambda^{2}$. This time scale depends both on the molecule-lead coupling and the lead hopping. On the other hand, the screening time $\tau_{\text {scr }} \sim 1 /|b|$ is a property of the lead only, and the ratio $\tau_{\text {rel }} / \tau_{\text {scr }} \sim(b / \lambda)^{2}$ is always larger than unity. If the interaction $U$ becomes comparable to or larger than $b$, then this analysis is not valid anymore. In Appendix $C$, we show that already at the $\mathrm{HF}$ level $U$ renormalizes the relaxation time according to $\tau_{\text {rel }} \sim \Gamma^{-1}(1-C U)^{2}$, where $C$ is a positive real constant weakly dependent on $U$ for small values of $U$. This is illustrated in the top panels of Fig. 5 where we display the real part of $G_{L L}^{>}(t, 0)$ for $b=-1.0$ and $\lambda^{r}=\lambda=-0.2$ at the HF and 2B levels. We see in both cases that by increasing the molecule-lead interaction $U$, we lower the relaxation time. The renormalization of the lead coupling (or the embedding SE) also affects the positions of the molecular quasiparticle levels. The renormalization leads to a small upward shift of the LUMO level and a downward shift of the HOMO level, i.e., a slight opening of the HOMO-LUMO gap. This is clearly visible in Fig. 5(a), where we see a slight increase in the frequency of the LUMO oscillation when we increase $U$. In Fig. 5(b) for 2B, on the other hand, we see a much more drastic decrease of the oscillation frequency due to the image charge effect, which HF fails to describe properly.

We note that the small upward shift within the HF approximation of the LUMO level with increasing $U$ can lead to an increase of the relaxation time when the level is close to the band edge. This is because the upward shift pushes the LUMO level close to the band edge where the imaginary part of the embedding self-energy decreases rapidly and compensates the renormalization introduced by the interaction $U$. In this case, the spectral peak describing the position and lifetime of the molecular quasiparticle level becomes also highly asymmetric and non-Lorenzian, which leads to a nonexponential decay of the Green's function in real time. These features are illustrated in Fig. 5(c), where we consider the case of lead hopping $b=-0.6$. The LUMO level for $U=0$ is located at 1 , which is close to the band edge of $2|b|=1.2$. An increase of $U$ to 1.3 pushes the level very close to the band edge and we then see a corresponding increase in relaxation time with a nonexponential decay. In the case of $2 \mathrm{~B}$ [Fig. 5(d)], the image charge effect pushes the level inward, away from the band edge, and we see that the relaxation time again decreases with increasing $U$. Comparing Fig. 5(d) to 5(b), we see further that increasing the lead hopping $b$ leads to a slight decrease of the image charge effect (frequency change for $U \neq 0$ ) and increase of the relaxation time, in agreement with the analysis of Sec. II B and the relation $\tau_{\text {rel }} \sim \Gamma^{-1} \sim|b| / \lambda^{2}$.

The difference between the $\mathrm{HF}$ and the correlated results in real time translates into a different spectral structure in frequency space. In Fig. 6, we display the quasiparticle spectral functions [see Eq. (20)] for the LUMO level $A_{L L}(\omega)$, corresponding to the Green's functions $G_{L L}^{>}(t, 0)$. This is done for the $2 \mathrm{~B}$ approximation using $b=-0.6$ and different values of $U$. For the uncontacted case, the $2 \mathrm{~B}$ result coincides with the GW result, as discussed in Sec. II C. The corresponding spectral function for $U=1.0$ is displayed in the left panel, while in the right panel we have $\lambda=-0.2$ and we plot the spectral function for different values of $U$. The very fast oscillations in the left panel are due to the finite-time interval in the Fourier transform. They are not present in the right panel due to damping of the Green's function in the contacted case. Aside from the main peak located at the electron affinity, we observe a shoulder of width $\Delta \approx 4|b|$ at higher energies. At finite hybridization $\lambda=-0.2$, this shoulder is smoother and partially merges with the main peak. The shoulder originates from the particle-hole continuum of excitations induced by the sudden addition of an electron to the LUMO state. They are the excitations that allow for the dynamical screening of the extra charge on the molecule. In mathematical terms, the shoulder arises by Fourier transforming the initial transient of the 2B curve in Figs. 4 and 5. Since no transient was observed in $\mathrm{HF}$, the HF spectral function will consist only of a main peak at the electron affinity. Both $2 \mathrm{~B}$ and $\mathrm{GW}$ incorporate the correct physics through the polarization diagram of Fig. 3(a), which nicely illustrates how an extra electron on the LUMO can excite a particle hole on the terminal site of the leads. For small hybridizations, the polarization is approximatively equal to the response function of Fig. 2, which explains the width $4|b|$ of the shoulder. We further see in the right panel of Fig. 6 that while the peak moves leftward with increasing $U$, the width of the plateau remains roughly constant at $4|b|$. The
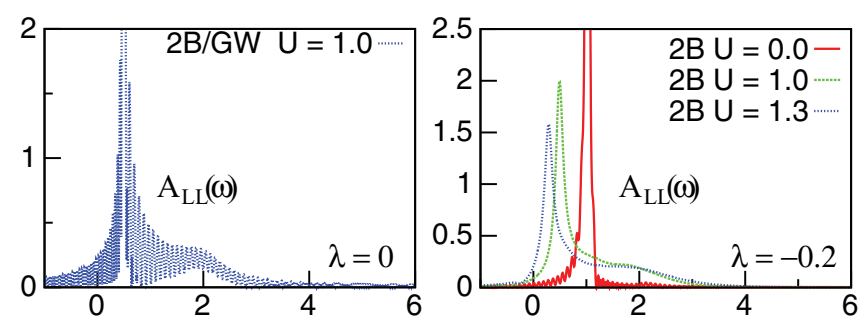

FIG. 6. (Color online) LUMO spectral function in the 2B approximation for $b=-0.6, U=1.0$, and $\lambda=0$ (left panel), $b=-0.6$, $\lambda=-0.2$, and $U=0,1.0,1.3$ (right panel). 
screening time is therefore independent of the molecule-lead interaction.

The message to take home is that the molecule-lead interaction has a large impact on the ratio $\tau_{\text {rel }} / \tau_{\text {scr }}$ and, in principle, can turn it to be smaller than one. These kinds of exotic situations would occur in leads with flat bands as, e.g., those modeled by Tasaki. ${ }^{54}$ In most metallic systems, this is not the case, and in the remainder of this paper we will study the regime $\tau_{\text {rel }} / \tau_{\text {scr }}>1$.

\section{B. Equilibrium spectral function}

In this section, we investigate the effects of screening on the spectral features of the molecule in equilibrium. We calculate the molecular spectral function $A_{\text {mol }}(\omega)$ as a sum of the projected spectral components $A_{i i}(\omega)$ as

$$
A_{\mathrm{mol}}(\omega)=-\frac{1}{\pi} \sum_{i=H, L} \operatorname{Im}\left[G_{i i}^{\mathrm{R}}(\omega)\right]
$$

for $U^{l}=\lambda^{l}=0$ and for zero and finite hybridization $\lambda^{r}=\lambda$ with the right lead. The results are shown in Fig. 7 for $\lambda=0$ and in Fig. 8 for $\lambda=-0.2$.

Let us start by analyzing the performance of the HF approximation. The first observation is that for $\lambda=0$, the HOMO-LUMO gap and the intensities of the peaks remain unchanged as the interaction strength $U^{r}=U$ increases. This can easily be understood from the explicit form of the HF HOMO and LUMO energies

$$
\begin{gathered}
\epsilon_{H}^{\mathrm{HF}}=\left(\epsilon_{H}-U\right)+n_{H} U_{0}+2 n_{L} U_{H L}+2 n_{1} U, \\
\epsilon_{L}^{\mathrm{HF}}=\left(\epsilon_{L}-U\right)+n_{L} U_{0}+2 n_{H} U_{H L}+2 n_{1} U .
\end{gathered}
$$

At half-filling, the average density $n_{1}=n_{1 r}$ of the right terminal site is $1 / 2$ and hence the dependence on $U$ cancels off. In the case of finite hybridization $\lambda=-0.2$ (Fig. 8), the HF peaks shift slightly outward and broaden due to the renormalization of the embedding self-energy [or, equivalently, the renormalization of the hybridization $\lambda \rightarrow \lambda+U G_{H 1}^{<}$(see Appendix C)]. It is then clear that for $\lambda=0$, the intensities

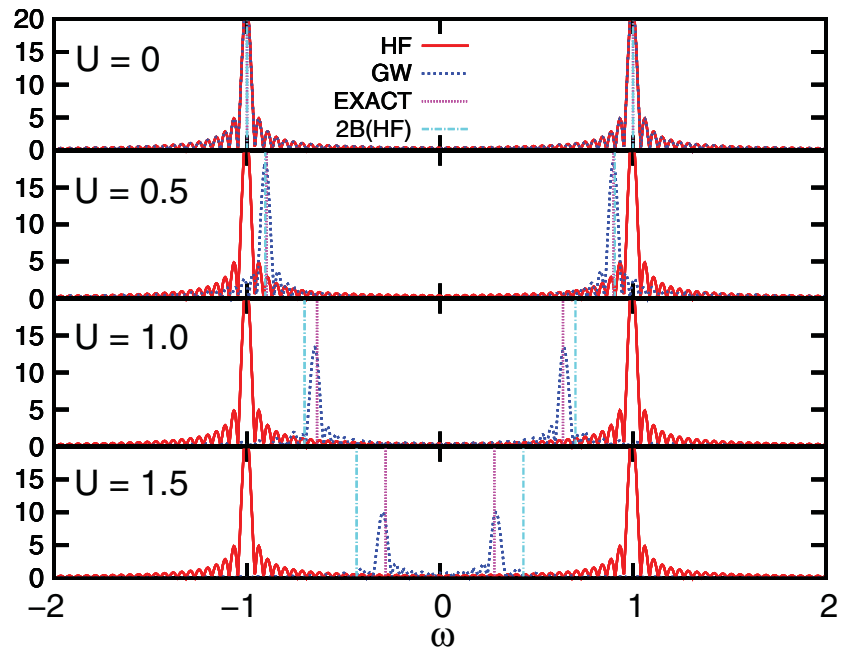

FIG. 7. (Color online) $\mathrm{HF}$ and $\mathrm{GW}$ equilibrium spectral functions $A_{\text {mol }}(\omega)$ for $\lambda=0, b=-1.0$, and $U=0,0.5,1.0,1.5$. The vertical lines indicate the exact and $2 \mathrm{Bs}(\mathrm{HF})$ peak positions.

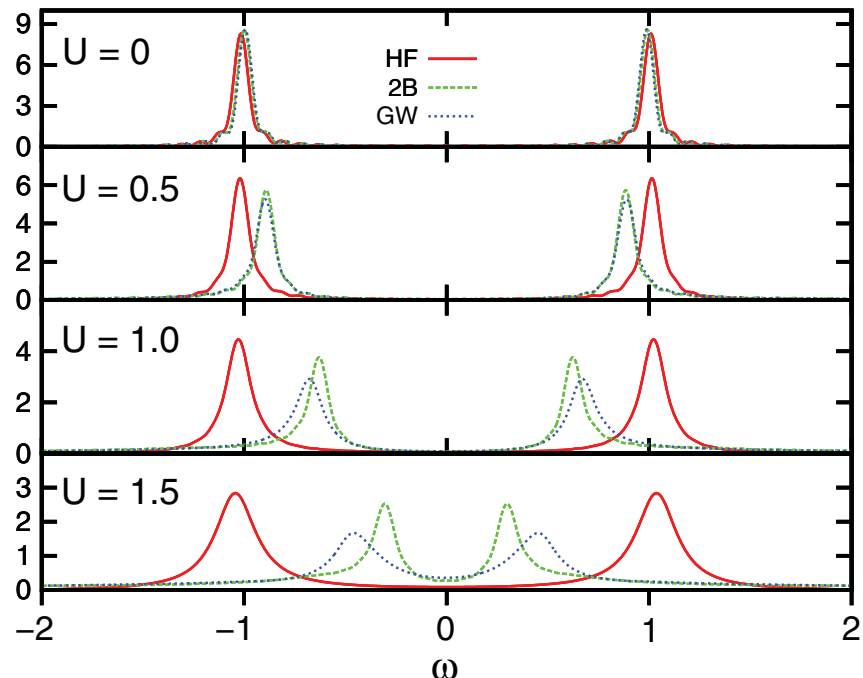

FIG. 8. (Color online) HF, 2B, and GW equilibrium spectral functions $A_{\mathrm{mol}}(\omega)$ for $\lambda=-0.2, b=-1.0$, and $U=0,0.5,1.0,1.5$.

do not change since $G_{H 1}=0$. Similar renormalization effects have been observed in Ref. 55. In the HF approximation, the self-energy of the $H / L$ levels couples only to the density at the terminal site of the lead and thus misses entirely the particle-hole coupling responsible for the screening.

The situation is radically different in the correlated $2 \mathrm{~B}$ and GW approximations. In both cases, the HOMO-LUMO gap, corresponding to the difference $A-I$ between the electron affinity and the ionization potential, narrows in agreement with the discussion of Sec. II B. The added (removed) electron and its image charge bind together, thereby decreasing (increasing) the addition (removal) energy. The stronger is the interaction $U$ and the larger is the gap reduction. In the $2 \mathrm{~B}$ and $\mathrm{GW}$ approximations, the added and removed electron couples not only to the density, but also to the particle-hole continuum of the lead. It is through this latter coupling that the charged system can lower its energy by exciting particles from occupied to unoccupied levels of the charge-neutral system. The resulting effect is to accumulate or deplete charge in the neighborhood of the terminal site, i.e., to screen the excess charge of the molecule. Note also that in the case $\lambda \neq 0$, the coupling to the particle-hole continuum provides an extra channel for quasiparticle scattering and induces quasiparticle broadening to the spectral peaks. The differences between the uncontacted and contacted spectral functions must be attributed to charge transfer processes and the consequent formation of image charges in the molecule. This molecular polarization effect was recently found to reduce the HOMO-LUMO gap even further. ${ }^{39}$

To assess the quality of the correlated approximations and the importance of self-consistency, we display in Fig. 7 the position of the exact $H / L$ peak (calculated from the Hellman-Feynman theorem) as well as the position of the peaks as obtained from a one-shot $2 \mathrm{~B}$ calculation with $\mathrm{HF}$ Green's function [denoted with $2 \mathrm{~B}(\mathrm{HF})$ ]. As can be seen from Fig. 7 , the GW results are in very good agreement with the 

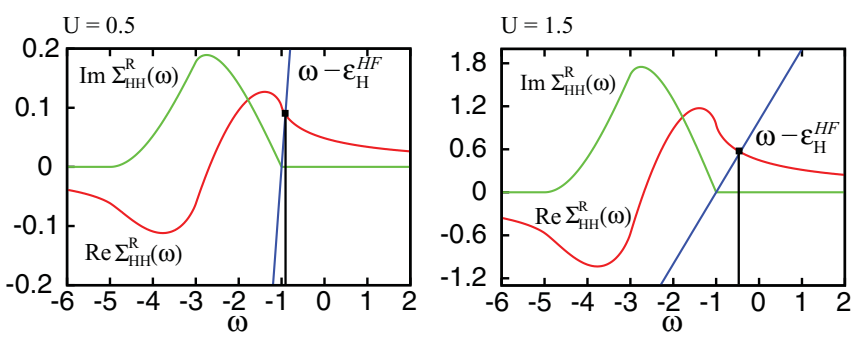

FIG. 9. (Color online) The real and imaginary components of the 2B(HF) self-energy for the molecular HOMO level with $U=0.5$ (left panel) and $U=1.5$ (right panel). The rest of the parameters are $U_{H L}=U_{0}=-b=1$ and $\lambda=0$.

exact ones. The position of the spectral peaks in the correlated approximations are obtained from the quasiparticle equation

$$
\omega-\epsilon_{i}^{\mathrm{HF}}-\operatorname{Re}\left\{\Sigma_{i i}^{\mathrm{R}}(\omega)\right\}=0,
$$

where $\Sigma_{i i}^{\mathrm{R}}(\omega)$ is the retarded many-body self-energy projected onto the $i=H, L$ molecular level. In Fig. 9, we display the graphical solution of Eq. (23) with $2 \mathrm{~B}(\mathrm{HF})$ self-energy and $i=H$. For this plot, we have chosen $U_{H L}=U_{0}=-b=1$ and $U=0.5$ (left panel) and $U=1.5$ (right panel). Already, one iteration of the self-consistency cycle captures the correct trend. The zero of Eq. (23) moves toward higher energies with increasing $U$. An analogous calculation for the LUMO level shows that the zero moves toward lower energy. In conclusion, the inclusion of polarization effects into the self-energy has two main effects in equilibrium: the redistribution of the spectral weight due to particle-hole excitations (satellite spectrum) and the collapse of the HOMO-LUMO gap. As we shall see, the situation is radically different out of equilibrium.

\section{QUANTUM TRANSPORT: SHORT-TIME DYNAMICS}

In order to investigate the short-time transport properties of the system of Fig. 1 , we consider $\lambda^{l}=\lambda^{r}=\lambda$ and $U^{l}=$ $U^{r}=U$. We will analyze the transient dynamics after the sudden switch-on of a bias $W^{l}=-W^{r}=W$ in the leads. Note from Eqs. (18) and (19) that the bias is applied also to the terminal (interacting) sites of the leads. We will refer to the left and right currents as the current flowing through the left and right interacting-noninteracting interfaces correspondingly. In all simulations, we set $\lambda=-0.2$ and hence work in the weak tunneling regime to highlight correlation effects.

\section{A. HF approximation}

In Figs. 10 and 11, we show the time-dependent currents [Figs. 10(a) and 11(a)], ground-state and nonequilibrium steady-state spectral functions [Figs. 10(b) and 11(b)], terminal site densities [Figs. 10(c) and 11(c)], and HOMO-LUMO densities [Figs. 10(d) and 11(d)] for the HF approximation with molecule-lead interaction $U=0.0,0.5,1.0$. In Fig. 10, we consider the "small" bias case $W^{l}=-W^{r}=0.8$ for which the equilibrium $H / L$ levels $\epsilon_{H / L}^{\mathrm{HF}}=\mp 1$ remain outside the bias window, while in Fig. 11 the bias is set to $W^{l}=-W^{r}=1.2$ so that the equilibrium $H / L$ levels lie inside the bias window.

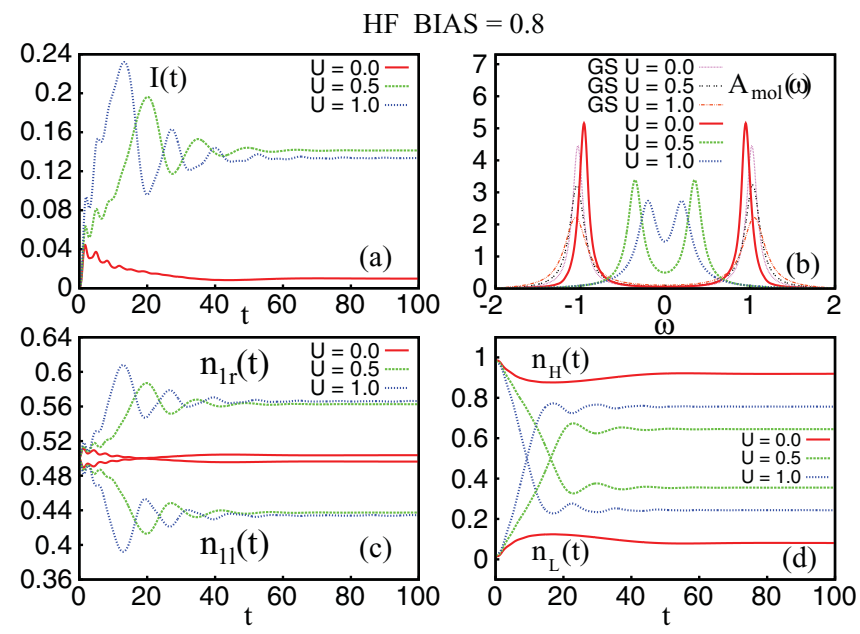

FIG. 10. (Color online) (a) Time-dependent right current for $U=0.0,0.5,1.0$. (b) Ground-state (GS) and steady-state spectral functions of Eq. (20). (c) Time-dependent densities $n_{1 r}(t)$ and $n_{1 l}(t)$ at the terminal sites. (d) Time-dependent HOMO and LUMO densities $n_{H}(t)$ and $n_{L}(t)$. In all the plots, the simulations have been performed within the HF approximation with bias $W^{l}=-W^{r}=0.8$.

For zero molecule-lead interaction $U=0$ and small bias, the current flowing through the system is almost zero [see Fig. 10(a)], in agreement with the fact that the $H / L$ levels are outside the bias window. A finite current instead sets in for large bias [see Fig. 11(a)]. The physics is here very similar to that of the noninteracting resonant transport regime. On the other hand, the current increases substantially at finite $U$ for small bias. Furthermore, by increasing $U$, the frequency and the amplitude of the oscillations in the current and density become larger. We recall that in the HF approximation, the equilibrium quantities are fairly independent of $U$. These

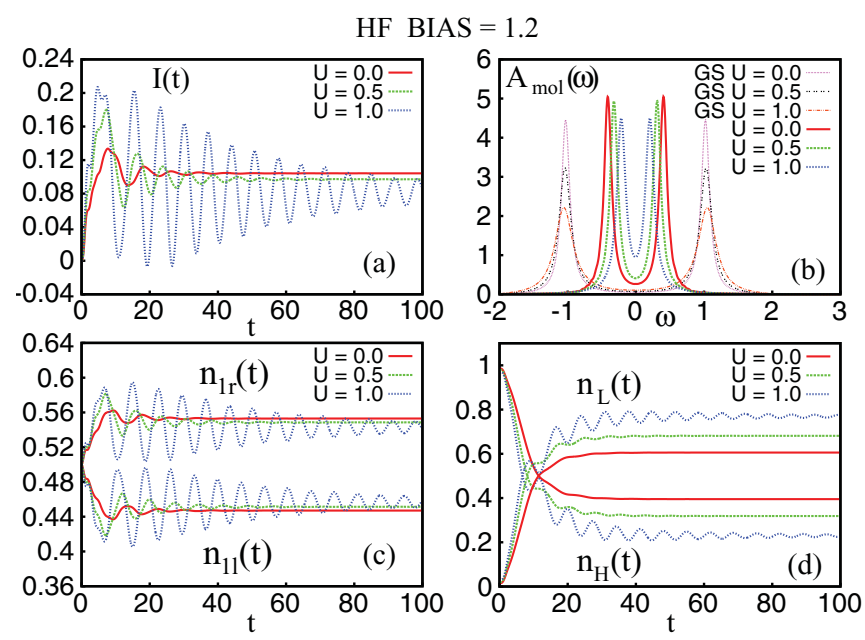

FIG. 11. (Color online) (a) Time-dependent right current for $U=0.0,0.5,1.0$. (b) Ground-state (GS) and steady-state spectral functions of Eq. (20). (c) Time-dependent densities $n_{1 r}(t)$ and $n_{1 l}(t)$ at the terminal sites. (d) Time-dependent HOMO and LUMO densities $n_{H}(t)$ and $n_{L}(t)$. In all the plots, the simulations have been performed within the HF approximation with bias $W^{l}=-W^{r}=1.2$. 
results show that at finite bias the situation is completely different.

To understand the differences between the equilibrium and the nonequilibrium cases, we observe that the gap in the nonequilibrium spectral function reduces considerably at finite $U$. For instance, Fig. 10(b) shows that for $U=0.5$ and 1.0, the $H / L$ levels have already entered the bias window $[-0.8,0.8]$. To trace back the physical origin of this effect, we write the HF energies of the $H / L$ levels as

$$
\begin{aligned}
& \epsilon_{H}^{\mathrm{HF}}=\epsilon_{H}-2 U+U_{0} n_{H}+2 U_{H L} n_{L}+2 U\left[n_{1 r}+n_{1 l}\right], \\
& \epsilon_{L}^{\mathrm{HF}}=\epsilon_{L}-2 U+U_{0} n_{L}+2 U_{H L} n_{H}+2 U\left[n_{1 r}+n_{1 l}\right],
\end{aligned}
$$

where we took into account that the molecule is now connected to both leads. The terms containing an explicit dependence on $U$ cancel off since the sum of the terminal site densities, $n_{1 r}(t)+n_{1 l}(t)$, remains roughly at its ground-state value during the entire time evolution [Fig. 10(c) and 11(c)]. Thus, it is not the lead polarization that affects the level positions, but rather the polarization of the molecular region, i.e., the difference $n_{H}-n_{L}$. Figures $10(\mathrm{~d})$ and 11(d) indicate that the molecular polarization increases as $U$ becomes large. This analysis shows that in the HF approximation, the reduction of the gap induced by $U$ has the same nature observed earlier ${ }^{16}$ and has nothing to do with the image charge effect. However, as we will see later, this effect already has a big impact on the resulting current-voltage characteristics.

The main frequency of the oscillations in the transient density and current originate from the electronic transitions from the left electrochemical potential $\mu^{l}=\epsilon_{F}+W^{l}$ to the LUMO level and also from the HOMO level to the right electrochemical potential $\mu^{r}=\epsilon_{F}+W^{r}$ (for the symmetric bias considered here, these transitions have the same energy). This can easily be verified by calculating the discrete Fourier transform of the transient current $I(\omega)$. In Fig. 12, we show
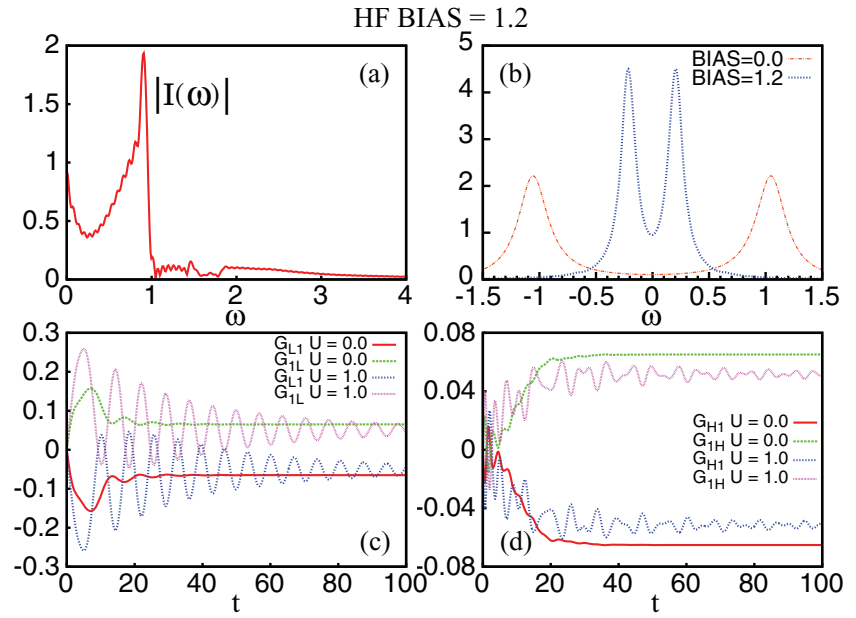

FIG. 12. (Color online) (a) Fourier transform of the transient current of Fig. 11 with $U=1.0$. (b) Ground-state and steadystate spectral functions $A_{\text {mol }}(\omega)$ for $U=1.0$. (c) Time-dependent density matrix components $G_{L, 1 l}\left(t, t^{+}\right)$and $G_{1 l, L}\left(t, t^{+}\right)$for $U=0.0$ and $U=1.0$ (d) HF time-dependent density matrix components $G_{H, 1 l}\left(t, t^{+}\right)$and $G_{1 l, H}\left(t, t^{+}\right)$for $U=0.0$ and 1.0. In all the plots, the simulations have been performed within the HF approximation with bias $W^{l}=-W^{r}=1.2$.
$I(\omega)$ for $U=1.0$ and the large bias case $W^{l}=-W^{r}=1.2$ [Fig. 12(a)] along with the ground-state and nonequilibrium steady-state spectral functions [Fig. 12(b)]. The Fourier transform $I(\omega)$ exhibits a sharp peak at $\omega \approx 1.0$ with a smearing toward lower frequencies down to $\omega \approx 0.2$. The smearing is a direct consequence of including the transient part of $I(t)$ in the Fourier transform. The value of $\epsilon_{H / L}^{\mathrm{HF}}$ is $\mp 1.0$ in equilibrium, while it is about $\mp 0.2$ at the steady state [see Fig. 12(b)]. As the HOMO-LUMO gap collapses, the transition energy between the left (right) electrochemical potential and the LUMO (HOMO) level changes from 0.2 to 1.0. The aforementioned smearing toward low frequency is the fingerprint of the dynamical renormalization of the transition frequency. Another consequence of the collapse of the steady-state gap with increasing $U$ is that $\epsilon_{H / L}^{\mathrm{HF}}$ moves further away from $\mu^{l / r}$ where the density of states has a square-root divergence (resonance condition). This is clearly illustrated in Fig. 11(b). The further away the levels are from resonance, the harder it is for electrons to tunnel, which in turn implies a larger oscillation amplitude and a smaller average current.

The transient oscillations are also visible in the off-diagonal components of the time-dependent density matrix $G_{i j}(t) \equiv$ $G_{i j}\left(t, t^{+}\right)$, which is displayed in Figs. 12(c) and 12(d) for $U=0$ and 1.0. The component $G_{L, 1 l}(t)$ and $G_{1 l, L}(t)$ oscillate with the same main frequency as the current and densities.
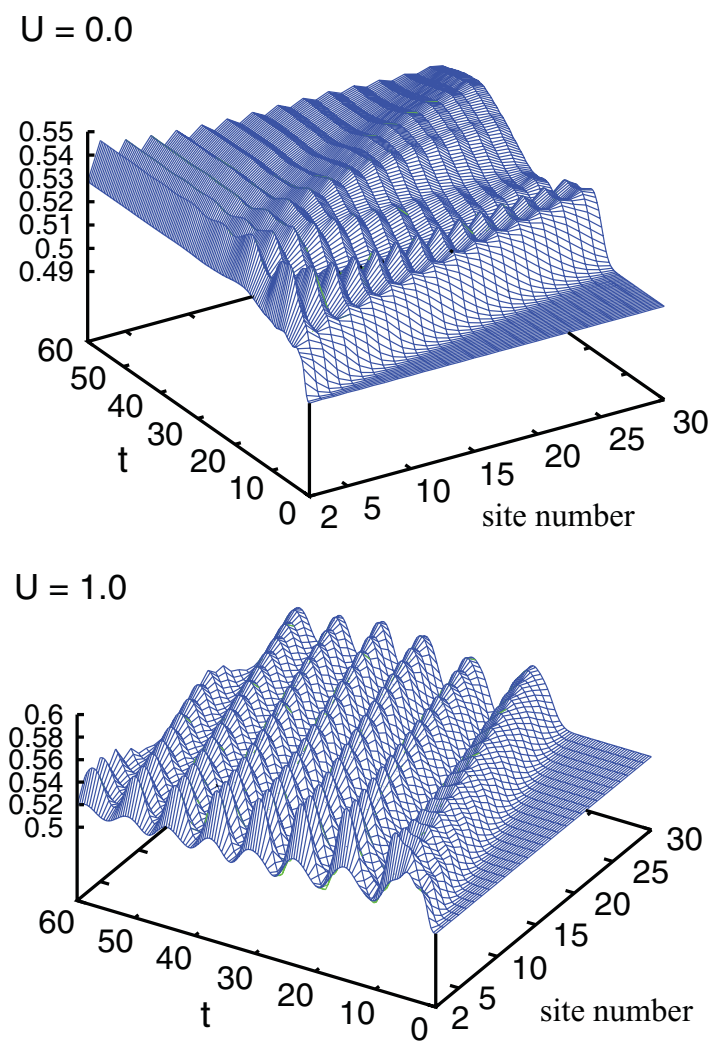

FIG. 13. (Color online) Time-dependent density in the noninteracting part of the right lead for $U=0.0$ (top panel) and $U=1.0$ (bottom panel). The simulations have been performed within the $\mathrm{HF}$ approximation with bias $W^{l}=-W^{r}=1.2$. Site number 2 corresponds to the first noninteracting site in the right lead. 
The same holds true for $G_{H, 1 r}(t)$ and $G_{1 r, H}(t)$ (not shown). On the contrary, $G_{H, 1 l}(t)$ and $G_{1 l, H}(t)$ have a very weak highfrequency component superimposed to the main frequency. Initially, the HOMO level is fully occupied and electronic transitions from $\mu^{l}$ to $\epsilon_{H}^{\mathrm{HF}}$ are blocked. Similarly, the LUMO level is initially empty, so there are no electronic transitions from $\mu^{r}$ to $\epsilon_{L}^{\mathrm{HF}}$. As the time passes, however, the HOMO occupation decreases while the LUMO occupation increases and these transitions become possible. They are located around 1.4 and 2.0 and can be seen in the Fourier transform of the current (the current is indeed given in terms of off-diagonal elements of the density matrix). Even though present, the transitions between the HOMO and the LUMO levels are extremely small since there is no direct hopping between the two levels.

The sudden switch-on of the bias gives rise to density shock waves in the leads with features similar to the density at the terminal sites. In Fig. 13, we show the transient dynamics of the HF density in the noninteracting part of the right lead for $U=0.0$ (top panel) and for $U=1.0$ (bottom panel) when the bias voltage is $W^{l}=-W^{r}=1.2$. The shock wave reaches site $j$ after a time $j / v_{F}$ where in our case the Fermi velocity $v_{F}=$ $2 b$. No matter how far site $j$ is, the density at this site exhibits damped oscillations, the initial amplitude and relaxation time of which is independent of $j$ and increases with $U$.

\section{B. Correlated approximations}

The inclusion of correlations changes considerably the physical picture. Let us focus on the large bias case $W^{l}=$ $-W^{r}=1.2$ and calculate the same quantities as in Fig. 11 but within the $2 \mathrm{~B}$ and $\mathrm{GW}$ approximations. The results are displayed in Figs. 14 and 15, respectively. The first important feature is that the relaxation time is much shorter than in the $\mathrm{HF}$ case due to the many-body broadening of the HOMO and LUMO levels [see Figs. 14(b) and 15(b)]. In the same figures, we also show the ground-state spectral function for the same values of $U$. As expected, the GS gap between the HOMO and LUMO peaks reduces with increasing $U$ due to the image charge effect. In the biased system for $U=0$, the bias-dependent gap closing ${ }^{9,16}$ brings the levels so close to each other that we can observe only one very broad peak. Interestingly and surprisingly, the effect of increasing $U$ in the biased system is to open the gap and to sharpen the spectral peaks. In the $2 \mathrm{~B}$ approximation with molecule-lead interaction $U=1.0$, the nonequilibrium steady-state gap is even larger than the ground-state gap. The GW approximation attenuates the gap opening compared to the $2 \mathrm{~B}$ approximation, but the sharpening of the peaks is well visible also in this case. The gap opening in the out-of-equilibrium system has never been reported before and, as we shall see below, has profound consequences on the $I-V$ curve.

\section{QUANTUM TRANSPORT: LONG-TIME DYNAMICS}

In this section, we investigate the effects of the image charge on the long-time dynamics of the lead-molecule-lead system within the HF, 2B, and GW approximations. As we shall see, a nontrivial post-transient dynamics develops at the HF level. The inclusion of correlations does always bring the system

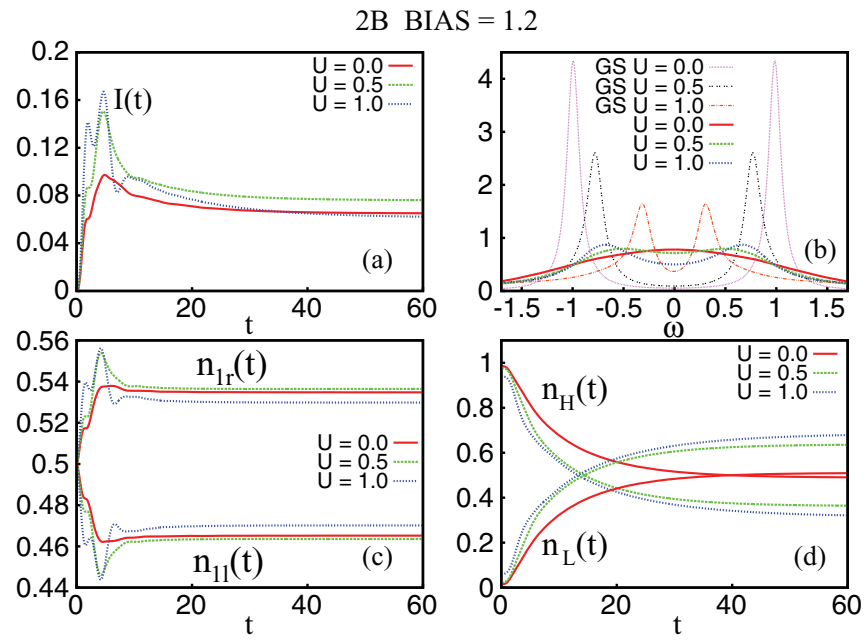

FIG. 14. (Color online) (a) Time-dependent right current for $U=0.0,0.5,1.0$. (b) Ground-state (GS) and steady-state spectral functions of Eq. (20). (c) Time-dependent densities $n_{1 r}(t)$ and $n_{1 l}(t)$ at the terminal sites. (d) Time-dependent HOMO and LUMO densities $n_{H}(t)$ and $n_{L}(t)$. In all the plots, the simulations have been performed within the $2 \mathrm{~B}$ approximation with bias $W^{l}=-W^{r}=1.2$.

in a steady-state regime. We will show how this regime is attained and calculate current and densities in the steady state for different bias voltages and molecule-lead interaction.

\section{A. HF approximation and post-transient dynamics}

We focus on the large bias regime and strong molecule-lead interaction $U=1.0$. In the previous section, we showed that current and densities seem to relax after the transient behavior induced by the sudden switch-on of a bias voltage. However, by extending further the propagation time window, something

$\mathrm{GW}$ BIAS $=1.2$
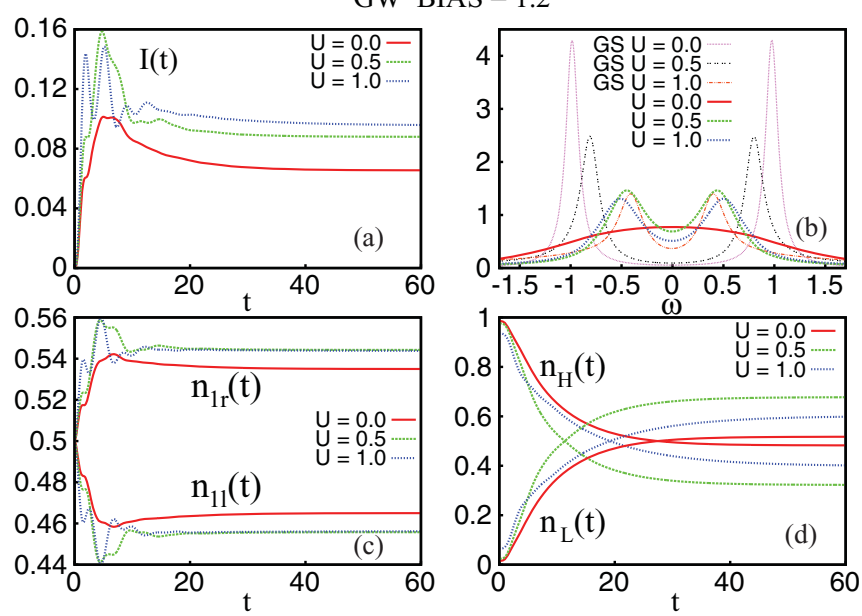

FIG. 15. (Color online) (a) Time-dependent right current for $U=0.0,0.5,1.0$. (b) Ground-state (GS) and steady-state spectral functions of Eq. (20). (c) Time-dependent densities $n_{1 r}(t)$ and $n_{1 l}(t)$ at the terminal sites. (d) Time-dependent HOMO and LUMO densities $n_{H}(t)$ and $n_{L}(t)$. In all the plots, the simulations have been performed within the $\mathrm{GW}$ approximation with bias $W^{l}=$ $-W^{r}=1.2$. 

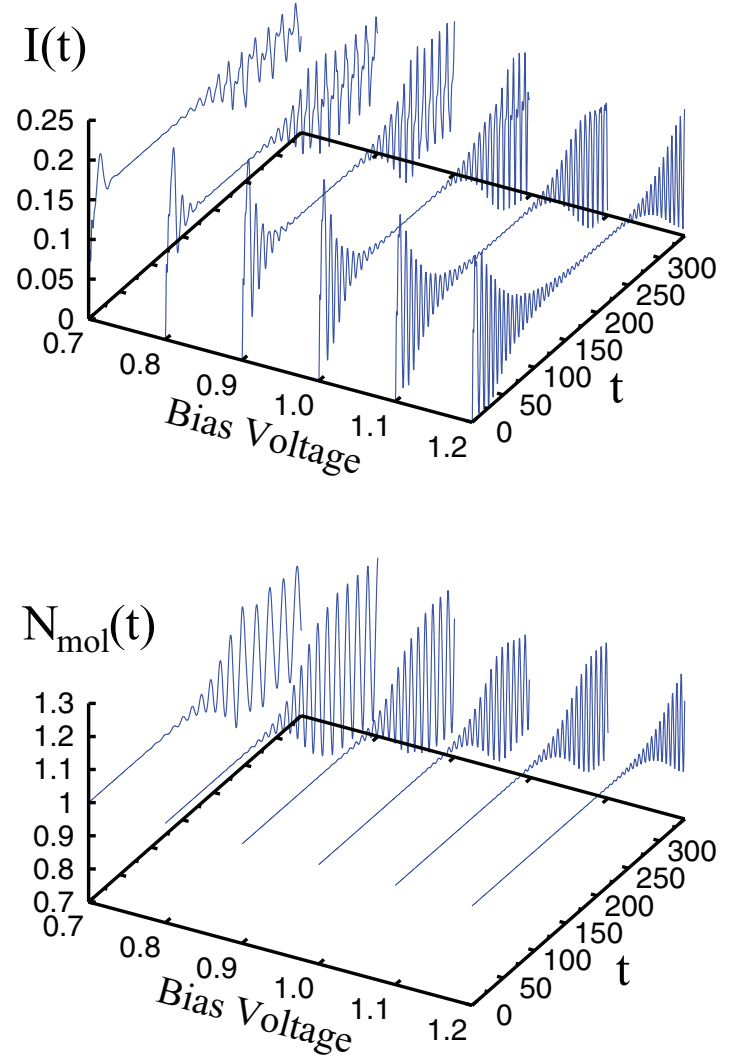

FIG. 16. (Color online) Time-dependent right current (top panel) and total number of particles in the molecule (bottom panel) for bias voltages in the range [0.7,1.2] and molecule-lead interaction $U=1.0$.

unexpected occurs. We find that the steady state is metastable, and oscillations with increasing amplitude develop to then stabilize in a periodic state. In Fig. 16, we display long-time simulations of the right current $I(t)$ (top panel) and the total number of particles in the molecule $N_{\text {mol }}(t)$ (bottom panel) for bias voltages in the range [0.7,1.2]. In this range, the equilibrium HOMO and LUMO levels lie in the bias window. The frequency of the oscillations increases as the bias voltage is increased, which is a clear indication that the dominant transitions are those between the leads and the molecular levels.

In Fig. 17, we display the time-dependent left and right currents [Fig. 17(a)] as well as the terminal-site densities [Fig. 17(c)] and molecular densities [Fig. 17(d)] for $W^{l}=$ $-W^{r}=0.8$. According to these results, the post-transient periodic state corresponds to a sequence of charge blockades with opposite sign of the electron-liquid acceleration (time derivative of the current) between two consecutive blockades. The oscillations are therefore due to a charge sloshing between the molecular levels and the terminal sites. The metastability of a steady-state solution in which current and densities are given by the average value of the time-dependent results is due to the combination of the constant flow of electrons from left to right and the self-consistent nature of the Hartree-Fock potential. Finally, we emphasized that the amplitude of the ac current superimposed to the dc current depends on where the current is measured.
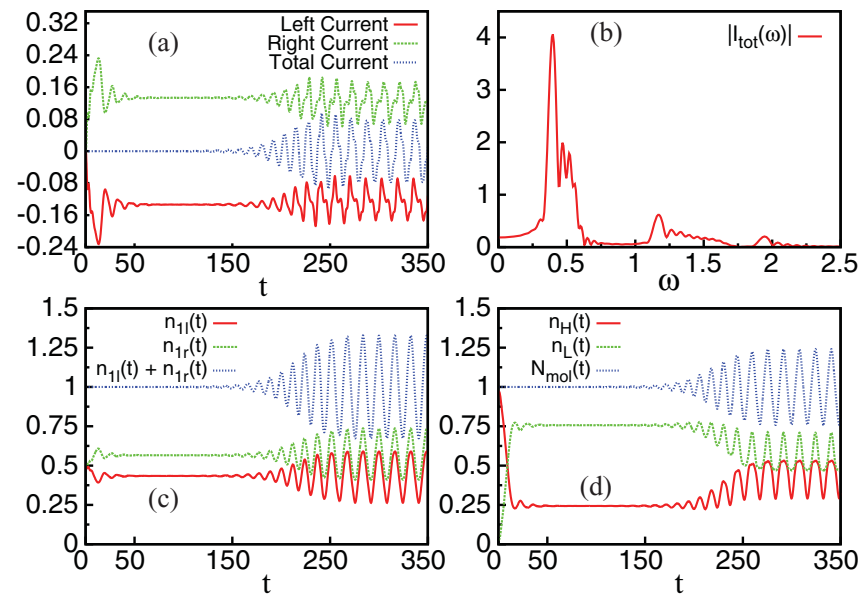

FIG. 17. (Color online) (a) Time-dependent left, right, and total currents. (b) Fourier transform of the total current. (c) Timedependent terminal site densities. (d) Time-dependent HOMO and LUMO densities and the total number of particles in the molecule. In all panels, $U=1.0$ and the bias voltage is $W^{l}=-W^{r}=0.8$.

In Fig. 17(b), we report the Fourier transfrom of the total current $I_{\text {tot }}(\omega)$. The main peak at $\omega \approx 0.4$ is smeared out toward higher frequencies up to $\omega \approx 0.6$, indicating the occurrence of electronic transitions between levels, the position of which changes dynamically in time. We also observe higher-frequency satellites around $\omega \approx 1.2,1.8$. These satellites occur exactly at the positions of odd harmonics of the main frequency. The absence of even harmonics is due to the fact that the external driving field is an odd function in space.

The persistent oscillatory behavior reported in this section is most likely an artifact of the HF approximation and, as we shall see in the next section, disappears in the $2 \mathrm{~B}$ and $\mathrm{GW}$ approximations. Within HF, the system knows only the instantaneous density and there is no damping mechanism to wash out the oscillations. These oscillations are sustained by the finite bias voltage and originate from the instantaneous Coulombic feedback.

\section{B. Steady-state properties: HF, 2B, and GW approximations}

In Fig. 18, we show the HF time-dependent currents and the resulting $I-V$ characteristic (bottom right panel) for different interaction strengths $U=0.0,0.5,1.0$. Since the HF currents for $U=1.0$ do not attain a steady state for large enough bias, the $I-V$ characteristic is in this case calculated with the dc part of the current (average value). The inclusion of the molecule-lead interaction deforms the $I-V$ characteristics dramatically. First, increasing the interaction strength, the threshold is shifted toward smaller bias values. Second, increasing the interaction strength up to $U=1.0$ gives rise to an extra step in the $I-V$ curve. The shift of the $I-V$ step toward smaller biases is related to the gap-closing mechanism, which in the $\mathrm{HF}$ approximation is entirely due to the intramolecular interactions $U_{0}$ and $U_{H L}$ (see Sec. IV A).

The extra step in the HF $I-V$ curve (bottom panel of Fig. 18) corresponds to a charged state of the molecule. In Fig. 19, we plot the number of particles (per spin) in the molecule $N_{\text {mol }}$ for interaction $U=1.0$. There exists a narrow window 

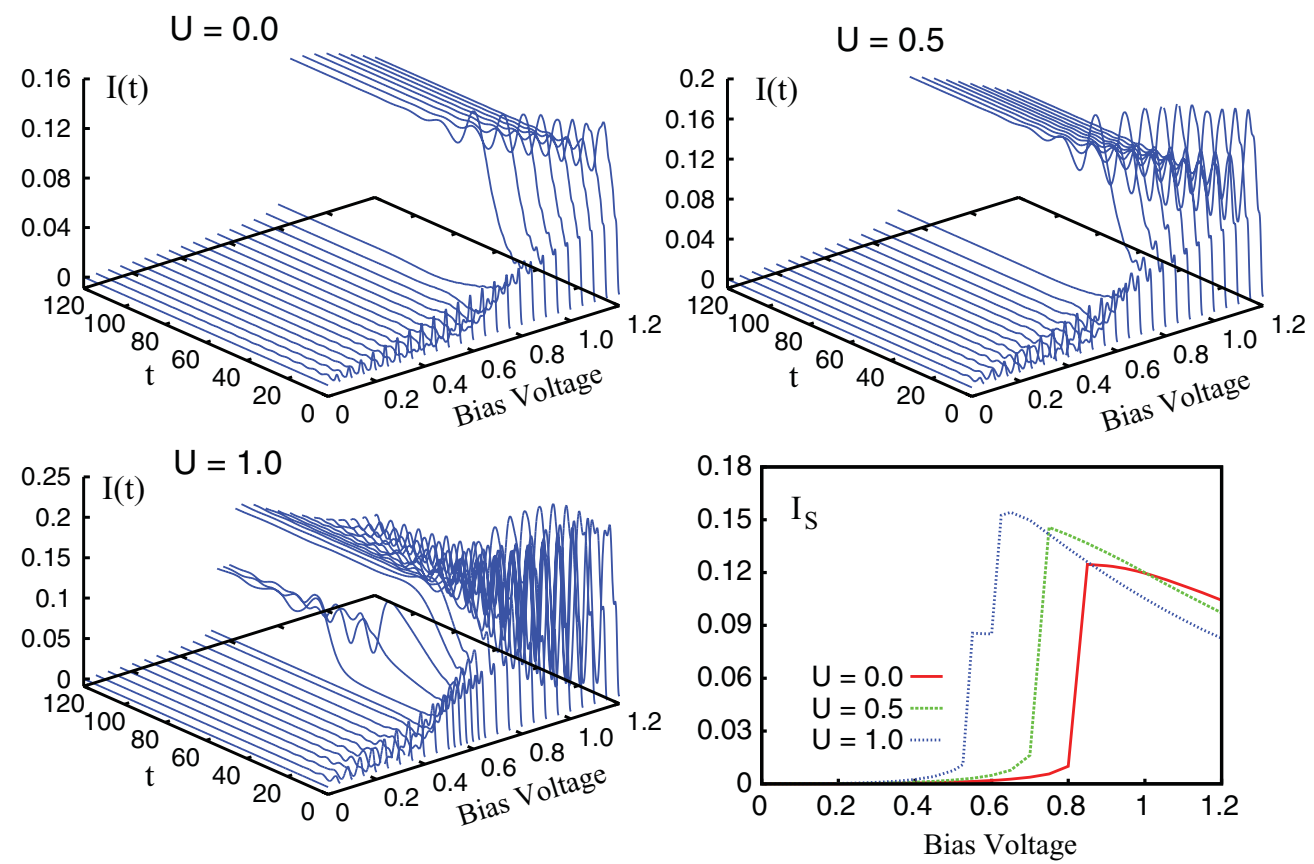

FIG. 18. (Color online) HF time-dependent right current for different interaction strengths $U=0,0.5$ (top left and right panels) and $U=1.0$ (bottom left panel). The $I-V$ curves extracted from the long-time limit are displayed in the bottom right panel.

of applied biases $W^{l}=-W^{r} \in[0.55,0.6]$ for which $N_{\text {mol }} \approx$ 1.35. We have also checked (not shown here) that this window can be extended by increasing the molecule-lead coupling $\lambda$. The excess molecular charge produces a Hartree barrier on the terminal sites, which prevents the current to increase (see plateau in the $I-V$ curve for $U=1$ ). As the bias becomes larger, electrons gain enough energy to overcome the barrier and the current increases again.

The excess charge on the molecule changes also the spectral function. In Fig. 20, we plot the full spectral function of the interacting region as well as the local spectral functions of the HOMO, LUMO, and the terminal sites in the ground and steady states for $U=1.0$ and for bias $W^{l}=-W^{r}=0.55$ within the HF approximation. The HF spectral function of the charged molecule exhibits two sharp structures close to the left and right band edges (they are separated by $W^{l}-W^{r}=1.1$ ). The induced Hartree barrier pushes electrons away from the terminal sites and gives rise to well-localized hole states. The

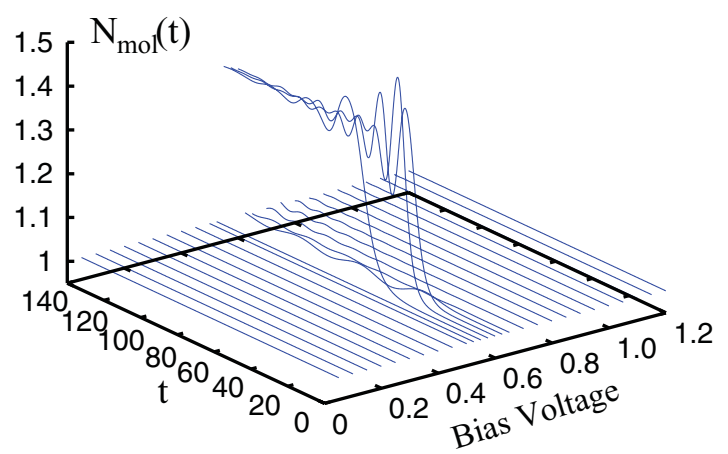

FIG. 19. (Color online) Time-dependent number of electrons on the molecule $N_{\text {mol }}(t)$ versus the applied bias voltage. HF approximation with $U=1.0$. structure of the peaks is indeed similar to that of a split-off state (antibound state), which forms in the presence of an external positive potential at the end site of a semi-infinite chain (see Appendix A). In our case, this potential is $v^{\alpha}=W^{\alpha}+v_{\mathrm{H}}$ with Hartree potential $v_{\mathrm{H}}=2 U\left(N_{\mathrm{mol}}-1\right) \approx 0.7$.

The formation of the additional step in the $I-V$ curve is probably another artifact of the HF approximation. In Figs. 21 and 22, we show the transient and steady-state currents for $U=$ $0,0.5$ and $U=1.0$ and bias voltage in the range $[0,1.2]$ within the $2 \mathrm{~B}$ and $\mathrm{GW}$ approximations. As for the HF approximation,
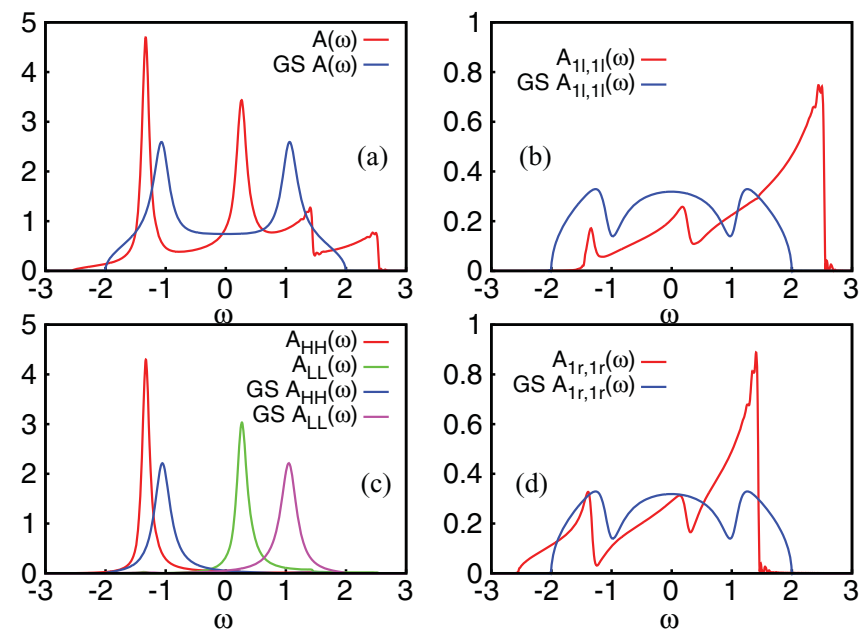

FIG. 20. (Color online) Ground-state (GS) and steady-state spectral functions in the HF approximation for $U=1.0$ and bias $W^{l}=$ $-W^{r}=0.55$. (a) Full spectral function of the interacting region. (b) Spectral function on the terminal site of the left lead. (c) HOMO and LUMO spectral functions. (d) Spectral function on the terminal site of the right lead. 

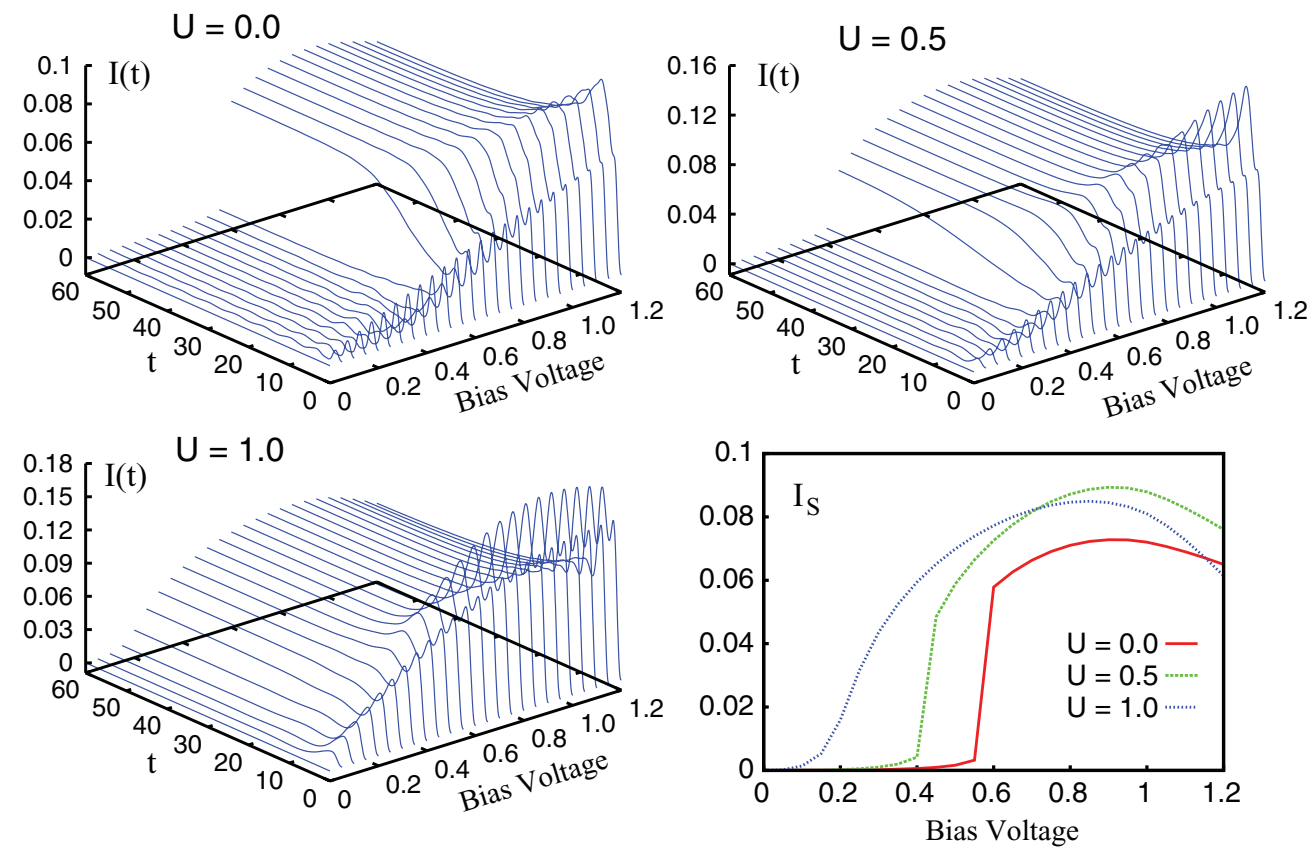

FIG. 21. (Color online) 2B time-dependent right current for different interaction strengths $U=0,0.5$ (top left and right panels) and $U=1.0$ (bottom left panel). The $I-V$ curves extracted from the long-time limit are displayed in the bottom right panel.

the onset of the current is shifted toward smaller bias values when $U$ increases. However, this effect is more pronounced in the $2 \mathrm{~B}$ and $\mathrm{GW}$ approximations, which properly incorporate dynamical polarization effects to account for the formation of the image charge. Another effect of correlations is to smoothen the onset, in agreement with the appearance of a particle-hole shoulder in the spectral function (see Sec. III A).

It is important to disentangle the scattering-induced broadening due to the intramolecular interactions $U_{0}$ and $U_{H L}$ from that due to the molecule-lead interaction $U$. In Fig. 23, we plot the full spectral functions of the interacting region within the $\mathrm{HF}, 2 \mathrm{~B}$, and $\mathrm{GW}$ approximations for two different values of $U=0$ and 1.0. For $U=0$ and within $2 \mathrm{~B}$ and $\mathrm{GW}$ there is a consistent broadening of the HOMO and LUMO peaks when these levels enter the bias window. This effect was reported previously in Refs. 9 and 17. However, for $U=1$, the $2 \mathrm{~B}$ and GW spectral functions do not get broader as they enter the bias window. The peaks preserve their shape and
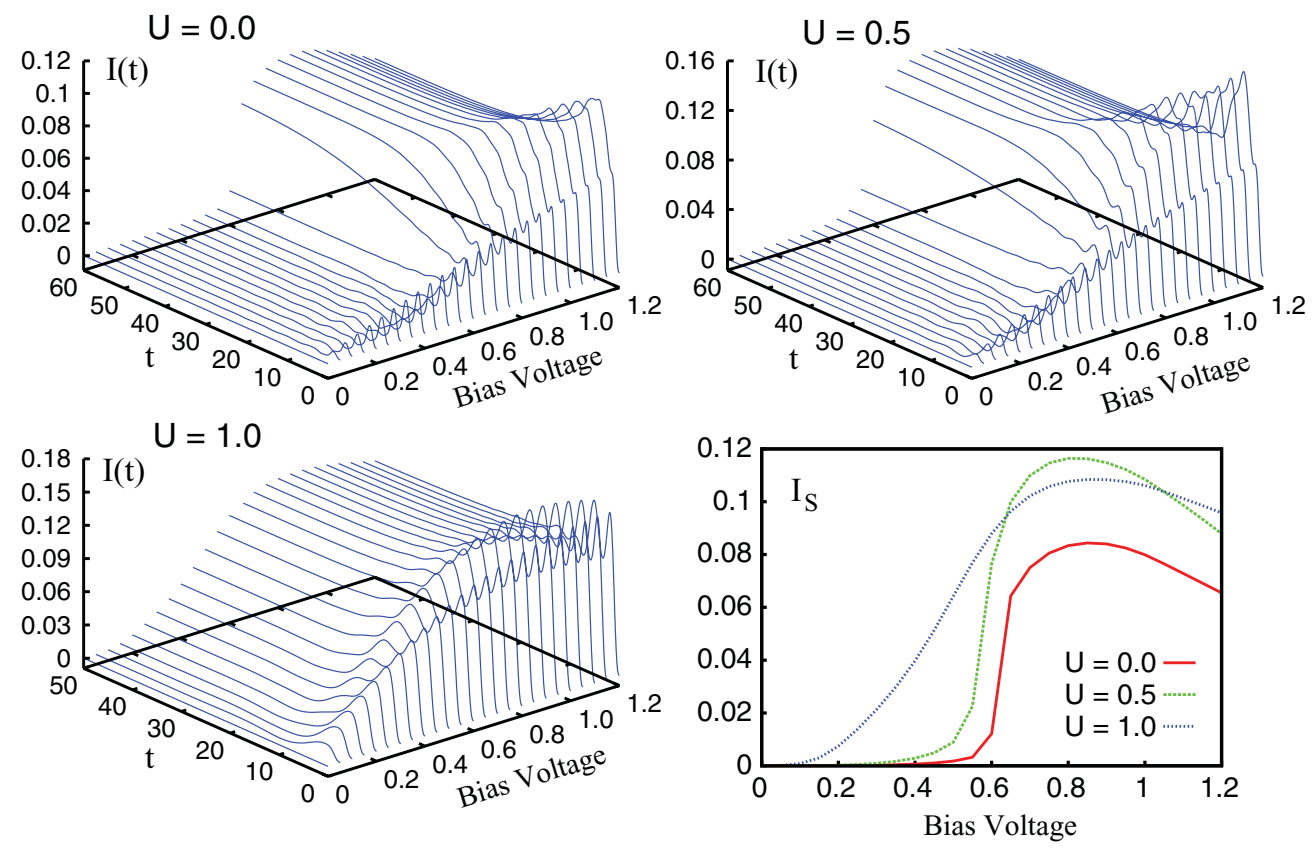

FIG. 22. (Color online) GW time-dependent right current for different interaction strengths $U=0,0.5$ (top left and right panels) and $U=1.0$ (bottom left panel). The $I-V$ curves extracted from the long-time limit are displayed in the bottom right panel. 

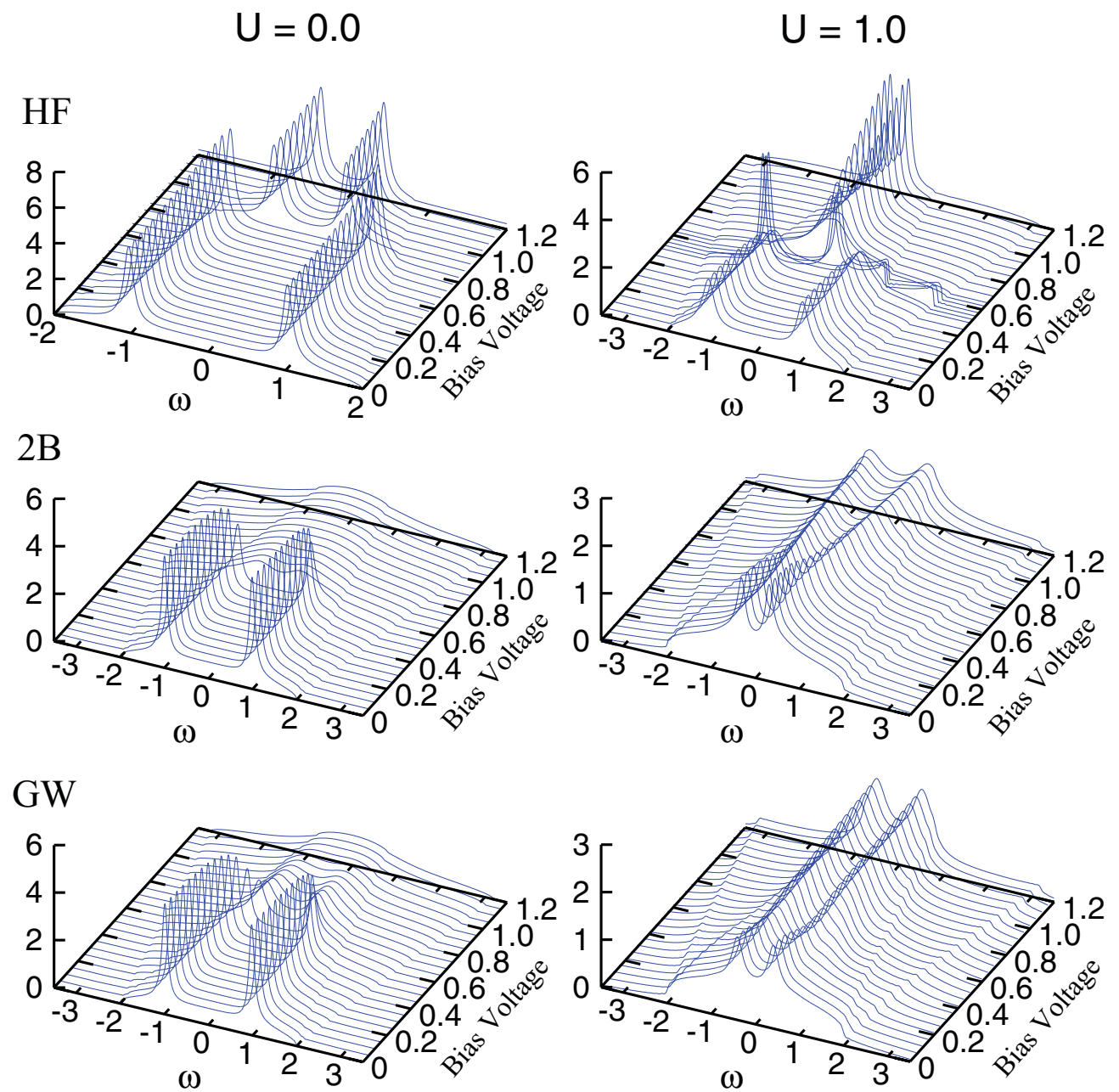

FIG. 23. (Color online) HF, 2B, and GW spectral functions for different bias voltage and lead-molecule interaction $U=0.0$ and 1.0 .

the HOMO-LUMO gap starts to open up. The molecule-lead interaction has an effect opposite to that of the intramolecular interaction on the broadening and the many-body shift of the spectral peaks. This is a very important result according to which image charge effects in the biased system contribute to lengthen the HOMO (LUMO) quasiparticle lifetimes and decrease (increase) the ionization potential (electron affinity).

\section{CONCLUSIONS}

In conclusion, we provided a thorough analysis of the effects of the dynamical formation of image charges at the interfaces between a molecule and the metallic leads under nonequilibrium conditions. The analysis has been carried out within the embedded Kadanoff-Baym method using fully self-consistent many-body approximations at the HF, 2B, and GW levels. The mean-field HF approximation fails to capture the polarization effects both in and out of equilibrium. As a consequence, the equilibrium molecular levels are not renormalized, while out of equilibrium, the renormalization is solely due to the intramolecular interactions. We pointed out that the shortcomings of the HF approximation are also at the origin of other unphysical effects. There exists a finite range of applied biases for which the molecule is artificially charged.
This causes a depletion of the electron density at the interfaces and prevents the current to increase as the bias becomes larger (plateau in the $I-V$ characteristic). Furthermore, for large enough bias and molecule-lead interaction, the molecular system does not relax in the long-time limit. We reported the occurrence of the undamped oscillations in current and densities. These oscillations correspond to a charge sloshing between the molecular levels and the terminal sites.

To cure the problems of the mean-field theory, we resorted to the $2 \mathrm{~B}$ and $\mathrm{GW}$ approximations. In both approximations, the self-energy contains polarization diagrams, which correctly account for the screening of the charged molecule and hence are suited to describe the formation of image charges. In all situations considered, we did not observe a plateau in the $I-V$ characteristic nor the absence of relaxation. An important finding of our analysis is that by increasing the molecule-lead interaction, the ratio between screening time and the relaxation time changes and the screening time is primarily determined by the properties of the lead. As expected, the 2B and GW equilibrium HOMO-LUMO gap closes when increasing the molecule-lead interaction. Thus, the onset of the current in the $I-V$ characteristic is shifted to lower biases as compared to a noninteracting or mean-field calculation. Another remarkable effect pertains the molecule 
spectral properties as a function of the applied bias. In equilibrium, the molecule-lead interaction is responsible for the reduction of the HOMO-LUMO gap and for a substantial redistribution of the spectral weight to the satellites induced by the electron correlations. By increasing the bias, the situation changes. For zero molecule-lead interaction, the HOMO and LUMO peak near each other and considerably broaden when they enter the bias window. The effect of the molecule-lead interaction is to keep the spectral peaks sharp and to open the HOMO-LUMO gap. This effect is therefore exactly the opposite of that generated by the intramolecular interactions. All this phenomenology clearly shows the importance of a proper description of electron correlations in time-dependent and steady-state quantum transport.

\section{ACKNOWLEDGMENTS}

The authors want to thank A. Stan and A.-M. Uimonen for fruitful discussions. We like to thank the Academy of Finland and the Vilho, Yrjö, and Kalle Väisälä foundation for financial support and CSC IT center for providing resources for scientific computing.

\section{APPENDIX A: OTHER EXACT RESULTS OF THE IMAGE CHARGE MODEL IN THE UNCONTACTED CASE}

In this appendix, we derive some simple analytic result for the ICM with $\lambda^{l}=\lambda^{r}=0, U^{l}=0$, and $U^{r}=U$. We will show that the main qualitative features of the system in equilibrium can be captured already by considering leads of finite length. Let us consider the molecule with an extra electron on the LUMO level and a right lead with only two sites. The extra electron induces an impuritylike potential $U$ on the terminal site, and the single-particle eigenvalues of the lead Hamiltonian are then given by $\varepsilon_{1,2}=\mp \sqrt{(U / 2)^{2}+b^{2}}$. Let us denote by $M=\mathrm{GS}^{+}$the molecular configuration with the extra electron. At half-filling, the right lead has two electrons and the eigenstates $\left|\mathrm{GS}^{+} s\right\rangle$ of Eq. (8) are displayed in Fig. 24. Their energy is $\mathcal{E}_{0}=2 \varepsilon_{1}, \mathcal{E}_{1}=\varepsilon_{1}+\varepsilon_{2}$, and $\mathcal{E}_{2}=2 \varepsilon_{2}$. In a similar manner, we can calculate the lead eigenenergies for the molecule with an electron less. The resulting ionization potential and electron affinity are $I=E_{4}-$ $E_{3}=\epsilon_{H}+U_{0}-2|b|+2 \sqrt{(U / 2)^{2}+b^{2}}$ and $A=E_{5}-E_{4}=$ $\epsilon_{L}+2 U_{H L}+2|b|-2 \sqrt{(U / 2)^{2}+b^{2}}$ [see Eqs. (12) and (13)]. These energies correspond to the renormalized energies of the HOMO and LUMO levels. We thus see that by increasing the Coulomb interaction $U$, the HOMO and LUMO levels approach each other, in agreement with the general result of Sec. II B.

The density at the terminal site is unity if the molecule is charge neutral. However, since the molecule with one electron more (less) induces an impuritylike potential $\mp U$, the terminal site density in this case changes according to

$$
n_{1}(u)=\int_{-\infty}^{\mu} A_{11}(\omega, u) d \omega
$$

where $A_{11}(\omega, u)=-\frac{1}{\pi} \operatorname{Im}\left[G_{11}^{R}(\omega, u)\right]$ is the spectral function projected on the terminal site with an impuritylike potential

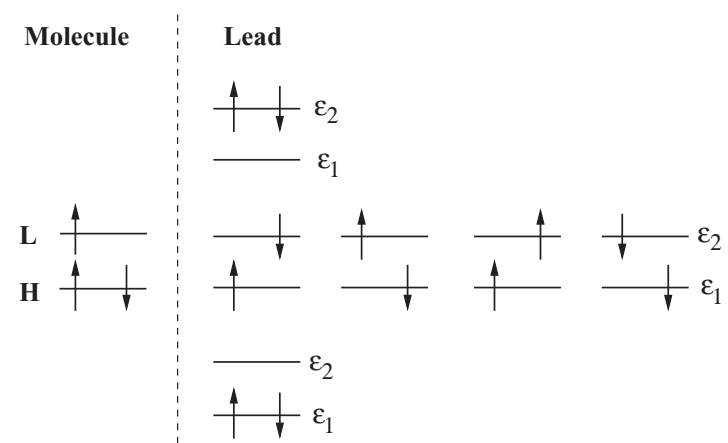

FIG. 24. Electronic configuration for a two-site lead. For an extra electron on the molecule, there are three energy eigenspaces for the lead. Note that the parallel-spin electron states do not contribute in the response properties since the bias preserves the spin orientation.

$u=\mp U$. The Green's function can be calculated explicitly from the Dyson equation and reads as

$$
G_{11}^{R}(\omega, U)=G_{11}^{0, R}(\omega) /\left[1-U G_{11}^{0, R}(\omega)\right] .
$$

Here, $G_{11}^{0, R}$ is the unperturbed retarded Green's function of the semi-infinite lead and it reads as

$$
G_{11}^{0, R}(\omega)=\frac{1}{2 b^{2}} \begin{cases}\left(\omega-\operatorname{sgn}(\omega) \sqrt{\omega^{2}-4 b^{2}}\right) & (|\omega|>2|b|), \\ \left(\omega-i \sqrt{4 b^{2}-\omega^{2}}\right) & (|\omega|<2|b|) .\end{cases}
$$

If $|U|$ exceeds the lead hopping $b$, a split-off state appears outside the energy continuum. This is illustrated in Fig. 25, where we plot the lead spectral function $A_{11}(\omega, U)$ for $U=$ $0,0.5,1.0,1.5$. This split-off state appears as a pole in the Green's function of Eq. (A2) with the energy

$$
\epsilon(U)=b\left[\frac{1+\left(\frac{U}{b}\right)^{2}}{\left(\frac{U}{b}\right)}\right] .
$$

Comparing the spectral structure of Fig. 25 with that of Fig. 20, we conclude that the extra step in the HF $I-V$ curve is due to the formation of a split-off state, which prevents the current to increase as the bias becomes larger.

\section{APPENDIX B: DENSITY RESPONSE FUNCTION}

We here calculate the density response function projected onto the terminal site of a semi-infinite TB chain relevant for the discussion of Sec. II B. For chains with $N_{\mathrm{ch}}$ sites, the single-particle eigenfunctions and eigenenergies of the system are $\psi_{k}(i)=(-1)^{i+1} \sqrt{\frac{2}{N_{\mathrm{ch}}+1}} \sin \left(\phi_{k} i\right)$ and $\epsilon_{k}=-2 b \cos \left(\phi_{k}\right)$, where $\phi_{k}=\frac{k \pi}{N_{\mathrm{ch}}+1}, k=1, \ldots, N_{\mathrm{ch}}$. By definition, the (retarded) density response function $\chi_{i j}(\omega)$ with site coordinates $(i, j)$ reads as

$$
\chi_{i j}(\omega)=2 \sum_{k l}\left(f_{k}-f_{l}\right) \frac{\psi_{k}^{*}(i) \psi_{l}(i) \psi_{k}(j) \psi_{l}^{*}(j)}{\omega-\left(\epsilon_{l}-\epsilon_{k}\right)+\mathrm{i} \eta},
$$

where, for zero temperature, $f_{k}=\theta\left(\mu-\epsilon_{k}\right)$ are the singleparticle occupations and $\eta$ is an infinitesimally small positive constant. By inserting the explicit form of the eigenfunctions and eigenvalues, changing the variables to $\bar{k}=k \pi /\left(N_{\mathrm{ch}}+1\right)$, 


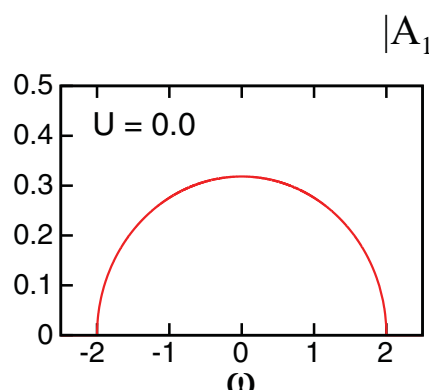

$\mathrm{A}_{11}(\omega)$
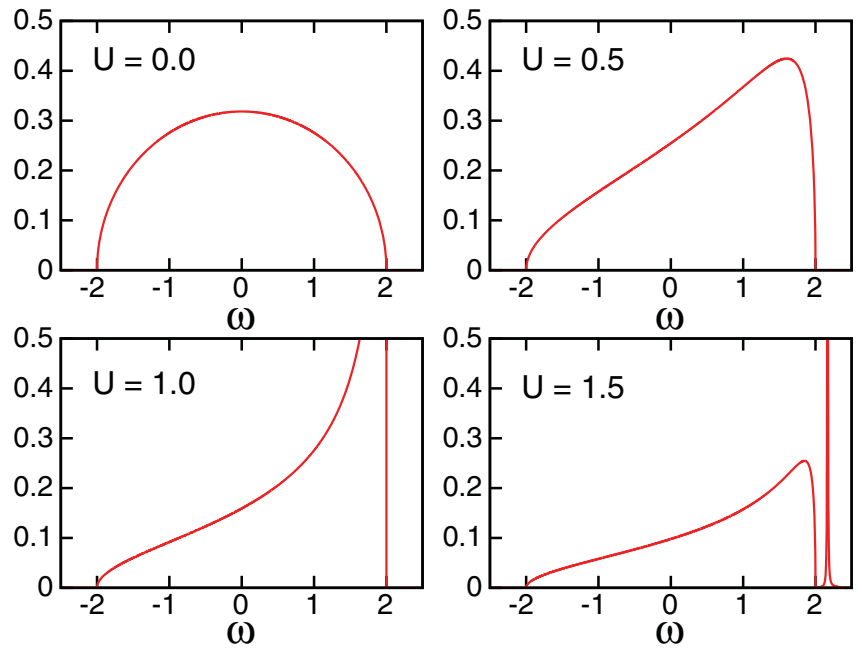

FIG. 25. (Color online) Formation of the split-off state (sharp peak in the bottom right panel) as $U$ increases. In all plots, $b=-1.0$.

and taking the $N_{\mathrm{ch}} \rightarrow \infty$ limit, we get for the $i=j=1$ component

$$
\chi_{11}(\omega)=\frac{8}{\pi^{2}} \int_{0}^{\pi} d \bar{k} \int_{0}^{\pi} d \bar{l} \frac{\left(f_{\bar{k}}-f_{\bar{l}}\right) \sin ^{2} \bar{k} \sin ^{2} \bar{l}}{\omega-2 b(\cos \bar{k}-\cos \bar{l})+\mathrm{i} \eta},
$$

where for the half-filled system here considered $f_{\bar{k}}=\theta\left(\frac{\pi}{2}-\right.$ $\bar{k})$. This expression can be simplified further by changing the variables to $x=\cos \bar{l}$ and $y=\cos \bar{k}$. The integral containing $f_{\bar{k}}$ becomes

$$
\begin{aligned}
\chi_{11}^{(1)}(\omega) & =\frac{8}{\pi^{2}} \int_{0}^{1} d y \int_{-1}^{1} d x \frac{\sqrt{1-y^{2}} \sqrt{1-x^{2}}}{\omega-2 b(y-x)+i \eta} \\
& =\Lambda^{(1)}(\omega)-\mathrm{i} \Delta^{(1)}(\omega),
\end{aligned}
$$

where $\Lambda^{(1)}(\omega)=\frac{1}{\pi} \mathcal{P} \int d \omega^{\prime} \frac{\Delta^{(1)}\left(\omega^{\prime}\right)}{\omega-\omega^{\prime}}$ is the real part and

$$
\begin{aligned}
\Delta^{(1)}(\omega)= & \frac{4}{\pi|b|} \int_{0}^{1} d y \sqrt{1-y^{2}} \sqrt{1-[y-\omega /(2 b)]^{2}} \\
& \times \theta[y-\omega /(2 b)+1] \theta\{1-[y-\omega /(2 b)]\}
\end{aligned}
$$

is the imaginary part. Similarly, one obtains the integral $\chi_{11}^{(2)}$ containing $f_{\bar{l}}$. The sum $\chi_{11}=\chi_{11}^{(1)}+\chi_{11}^{(2)}$ can now easily be calculated numerically.

\section{APPENDIX C: EXPLAINING THE LEVEL BROADENING IN THE HF APPROXIMATION}

In this Appendix, we show that the molecule-lead interaction in the presence of a finite hybridization renormalizes the embedding self-energy already in the HF approximation, thus explaining the broadening of the HF peaks in Fig. 8. Let us denote simply by $G$ and $\Sigma$ the retarded components of the Green's function and self-energy, respectively. For simplicity, we take $\lambda^{l}=U^{l}=0$ and $\lambda^{r}=\lambda, U^{r}=U$ and we denote by 1 the terminal site of the right lead. We start from the Dyson equation for $G(\omega)$ :

$$
\left(\omega-h-\Sigma^{\mathrm{HF}}\right) G(\omega)=1,
$$

where, in accordance with the notation of Sec. II C, $h$ is the Hamiltonian in the one-particle Hilbert space and has the structure

$$
h=\left(\begin{array}{ccc}
\epsilon_{H} & 0 & h_{H, r} \\
0 & \epsilon_{L} & h_{L, r} \\
h_{r, H} & h_{r, L} & h_{r, r}
\end{array}\right) .
$$

Here, $h_{r, r}$ is the tridiagonal matrix that describes the right lead with matrix elements $b$ on the upper and lower diagonals and zero otherwise, while $h_{i, r}$, with $i=H, L$, is the rectangular matrix whose only nonvanishing entry is $\left(h_{i, r}\right)_{i, 1}=\lambda$. By projecting the Dyson equation onto $H H$ and $r, H$ we find

$$
\begin{aligned}
\left(\omega-\epsilon_{H}-\Sigma_{H H}^{\mathrm{HF}}\right) G_{H H}(\omega) & =1+\left[h_{H, r}+\Sigma_{H, r}^{\mathrm{HF}}\right] G_{r, H}(\omega), \\
\left(\omega-h_{r, r}-\Sigma_{r, r}^{\mathrm{HF}}\right) G_{r, H}(\omega) & =\left[h_{r, H}+\Sigma_{r, H}^{\mathrm{HF}}\right] G_{H H}(\omega) .
\end{aligned}
$$

By solving the second equation for $G_{r, H}$ and inserting the result in the first equation, we obtain the following solution for $G_{H H}$ :

$$
G_{H H}(\omega)=\frac{1}{\omega-\epsilon_{H}-\Sigma_{H H}}
$$

with

$$
\Sigma_{H H}=\Sigma_{H H}^{\mathrm{HF}}+\left(\lambda+\Sigma_{H 1}^{\mathrm{HF}}\right) \tilde{G}_{11}(\omega)\left(\lambda+\Sigma_{1 H}^{\mathrm{HF}}\right) .
$$

In the above equation, $\tilde{G}_{11}$ is the $(1,1)$ matrix element of the Green's function of the uncontacted system $(\lambda=0)$ with the same HF self-energy, i.e., $\tilde{G}_{r, r}=1 /\left(\omega-h_{r, r}-\Sigma_{r, r}^{\mathrm{HF}}\right)$. Note that the only nonvanishing entry of the self-energy in the lead is $\left(\Sigma_{r, r}^{\mathrm{HF}}\right)_{11}=\Sigma_{11}^{\mathrm{HF}}$. Next, we observe that the nonlocal HF self-energy can be written as

$$
\Sigma_{1 H}^{\mathrm{HF}}=i U \int_{-\infty}^{\mu} \frac{d \omega}{2 \pi}\left(-2 i \operatorname{Im}\left[G_{1 H}(\omega)\right]\right),
$$

and similarly for $\Sigma_{1 H}^{\mathrm{HF}}$. From the projected Dyson equation, we have

$$
G_{1 H}(\omega)=\tilde{G}_{11}(\omega)\left(\lambda+\Sigma_{1 H}^{\mathrm{HF}}\right) G_{H H}(\omega) .
$$

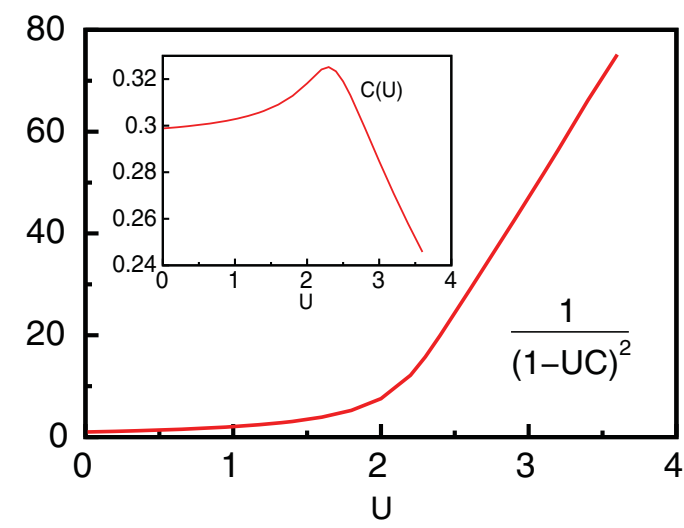

FIG. 26. (Color online) The value of the renormalization constant as a function of $U$ for $b=-1.0$ and $\lambda=-0.2$. The inset shows the dependence of the factor $C$ as a function of $U$. 
For the equilibrium system, we can always choose the $\mathrm{HF}$ orbital to be real valued and therefore $\operatorname{Im}\left[\Sigma_{1 H}^{\mathrm{HF}}\right]=0$. Then, by inserting Eq. (C6) into (C5) and solving for $\Sigma_{1 H}^{\mathrm{HF}}$, we find

$$
\Sigma_{1 H}^{\mathrm{HF}}=\lambda \frac{U C}{1-U C}
$$

where

$$
C=2 \int_{-\infty}^{\mu} \frac{d \omega}{2 \pi} \operatorname{Im}\left[\tilde{G}_{11}(\omega) G_{H H}(\omega)\right] .
$$

This result together with its analogous for $\Sigma_{H 1}^{\mathrm{HF}}$ allows us to cast the self-energy in Eq. (C4) in the form

$$
\Sigma_{H H}=\Sigma_{H H}^{\mathrm{HF}}+\left(\frac{1}{1-U C}\right)^{2} \Sigma_{H H}^{\mathrm{em}}(\omega),
$$

where $\Sigma_{H H}^{\mathrm{em}}(\omega)=\lambda^{2} \tilde{G}_{11}(\omega)$ is the embedding self-energy of the noninteracting system. Thus, the molecule-lead interaction renormalizes the embedding self-energy and increases the broadening of the HF spectral peaks. The value of the constant $C$ in Eq. (C8) can be determined numerically. In Fig. 26, we display $(1-C U)^{-2}$ and $C$ (inset) as a function of $U$. We see that $C$ is roughly constant for small $U$.
${ }^{1}$ M. A. Reed, C. Zhou, C. J. Muller, T. P. Burgin, and J. M. Tour, Science 278, 252 (1997).

${ }^{2}$ R. H. M. Smit, Y. Noat, C. Untiedt, N. D. Lang, M. C. van Hemert, and J. M. Ruitenbeek, Nature (London) 419, 906 (2002).

${ }^{3}$ Y. Meir and N. S. Wingreen, Phys. Rev. Lett. 68, 2512 (1992).

${ }^{4}$ J. Taylor, H. Guo, and J. Wang, Phys. Rev. B 63, 121104 (2001).

${ }^{5}$ M. Brandbyge, J. L. Mozos, P. Ordejon, J. Taylor, and K. Stokbro, Phys. Rev. B 65, 165401 (2002).

${ }^{6}$ A. R. Rocha, V. M. Garcia-Suarez, S. Bailey, C. Lambert, J. Ferrer, and S. Sanvito, Phys. Rev. B 73, 085414 (2006).

${ }^{7}$ D. M. Cardamone, C. A. Stafford, and S. Mazumdar, Nano Lett. 6, 2422 (2006)

${ }^{8}$ P. Darancet, A. Ferretti, D. Mayou, and V. Olevano, Phys. Rev. B 75, 075102 (2007).

${ }^{9}$ K. S. Thygesen, Phys. Rev. Lett. 100, 166804 (2008).

${ }^{10}$ J. P. Bergfield and C. A. Stafford, Phys. Rev. B 79, 245125 (2009).

${ }^{11}$ H. Ness, L. K. Dash, and R. W. Godby, Phys. Rev. B 82, 085426 (2010).

${ }^{12}$ V. Moldoveanu, H. D. Cornean, and C.-A. Pillet, Phys. Rev. B 84, 075464 (2011).

${ }^{13}$ M. Knap, W. von der Linden, and E. Arrigoni, Phys. Rev. B 84, 115145 (2011).

${ }^{14}$ A.-P. Jauho, N. S. Wingreen, and Y. Meir, Phys. Rev. B 50, 5528 (1994).

${ }^{15}$ G. Stefanucci and C-O. Almbladh, Phys. Rev. B 69, 195318 (2004); Europhys. Lett. 67, 14 (2004).

${ }^{16}$ P. Myöhänen, A. Stan, G. Stefanucci, and R. van Leeuwen, Europhys. Lett. 84, 67001 (2008).

${ }^{17}$ P. Myöhänen, A. Stan, G. Stefanucci, and R. van Leeuwen, Phys. Rev. B 80, 115107 (2009).

${ }^{18}$ P. Myöhänen, A. Stan, G. Stefanucci, and R. van Leeuwen, J. Phys.: Conf. Ser. 220, 012017 (2010).

${ }^{19}$ V. Moldoveanu and B. Tanatar, Europhys. Lett. 86, 67004 (2009).

${ }^{20}$ Y. Tomita, T. Nakayama, and H. IshiiJ. Surf. Sci. Nanotech.7, 606 (2009).

${ }^{21}$ V. Moldoveanu, A. Manolescu, and V. Gudmundsson, Phys. Rev. B 82, 085311 (2010).

${ }^{22}$ R. Baer, S. I. T. Seideman, and D. Neuhauser, J. Chem. Phys. 120, 3387 (2004).

${ }^{23}$ S. Kurth, G. Stefanucci, C.-O. Almbladh, A. Rubio, and E. K. U. Gross, Phys. Rev. B 72, 035308 (2005).
${ }^{24}$ X. Zheng, F. Wang, C. Y. Yam, Y. Mo, and G. H. Chen, Phys. Rev. B 75, 195127 (2007).

${ }^{25}$ P. Bokes, F. Corsetti, and R. W. Godby, Phys. Rev. Lett. 101, 046402 (2008).

${ }^{26}$ G. Stefanucci, S. Kurth, A. Rubio, and E. K. U. Gross, Phys. Rev. B 77, 075339 (2008).

${ }^{27}$ X. Zheng, G. Chen, Y. Mo, S. Koo, H. Tian, C. Yam, and Y. Yan, J. Chem. Phys. 133, 114101 (2010).

${ }^{28}$ M. Puig von Friesen, C. Verdozzi, and C.-O. Almbladh, Phys. Rev. B 82, 155108 (2010).

${ }^{29}$ J. S. Evans and T. Van Voorhis, Nano Lett. 9, 2671 (2009).

${ }^{30}$ K. Varga, Phys. Rev. B 83, 195130 (2011).

${ }^{31}$ G. Cohen and E. Rabani, Phys. Rev. B 84, 075150 (2011).

${ }^{32}$ E. C. Cuansing and G. Liang, J. Appl. Phys. 110, 083704 (2011).

${ }^{33}$ E. Perfetto, G. Stefanucci, and M. Cini, Phys. Rev. Lett. 105, 156802 (2010).

${ }^{34}$ D. Bohr and P. Schmitteckert, Phys. Rev. B 75, 241103 (2007).

${ }^{35}$ L. Borda, K. Vladár, and A. Zawadowski, Phys. Rev. B 75, 125107 (2007).

${ }^{36}$ F. Elste, D. R. Reichman, and A. J. Millis, Phys. Rev. B 83, 245405 (2011).

${ }^{37}$ E. Perfetto, G. Stefanucci, and M. Cini, e-print arXiv:1110.5161.

${ }^{38}$ J. B. Neaton, M. S. Hybertsen, and S. G. Louie, Phys. Rev. Lett. 97, 216405 (2006).

${ }^{39}$ K. S. Thygesen and A. Rubio, Phys. Rev. Lett. 102, 046802 (2009).

${ }^{40}$ J. M. Garcia-Lastra, C. Rostgaard, A. Rubio, and K. S. Thygesen, Phys. Rev. B 80, 245427 (2009).

${ }^{41}$ K. Kaasbjerg and K. Flensberg, Nano Lett. 8, 3809 (2008).

${ }^{42}$ K. Kaasbjerg and K. Flensberg, Phys. Rev. B 84, 115457 (2011).

${ }^{43}$ N. E. Dahlen and R. van Leeuwen, Phys. Rev. Lett. 98, 153004 (2007).

${ }^{44}$ K. Balzer, M. Bonitz, R. van Leeuwen, A. Stan, and N. E. Dahlen, Phys. Rev. B 79, 245306 (2009).

${ }^{45}$ K. Balzer, S. Bauch, and M. Bonitz, Phys. Rev. A 82, 033427 (2010).

${ }^{46}$ M. Puig von Friesen, C. Verdozzi, and C.-O. Almbladh, Phys. Rev. Lett. 103, 176404 (2009). 
${ }^{47}$ M. Puig von Friesen, C. Verdozzi, and C.-O. Almbladh, Phys. Rev. B 82, 155108 (2010).

${ }^{48}$ L. P. Kadanoff and G. Baym, Quantum Statistical Mechanics (Benjamin, New York, 1962).

${ }^{49}$ G. Baym, Phys. Rev. 127, 1391 (1962).

${ }^{50}$ A.M. Uimonen, E. Khosravi, A. Stan, G. Stefanucci, S. Kurth, R. van Leeuwen, and E.K.U. Gross, Phys. Rev. B 84, 115103 (2011).
${ }^{51}$ F. Heidrich-Meisner, A. E. Feiguin, and E. Dagotto, Phys. Rev. B 79, 235336 (2009).

${ }^{52}$ R. P. Feynman, Phys. Rev. 56, 340 (1939).

${ }^{53}$ A. Stan, N. E. Dahlen, and R. van Leeuwen, J. Chem. Phys. 130, 224101 (2009).

${ }^{54}$ H. Tasaki, Prog. Theor. Phys. 99, 489 (1998).

${ }^{55}$ M. Sade, Y. Weiss, M. Goldstein, and R. Berkovits, Phys. Rev. B 71, 153301 (2005) 